\title{
Will Automated Vehicles Negatively Impact Traffic Flow?
}

\author{
S. C. Calvert, W. J. Schakel, and J. W. C. van Lint \\ Department of Transport \& Planning, Delft University of Technology, Delft, Netherlands \\ Correspondence should be addressed to S. C. Calvert; s.c.calvert@tudelft.nl
}

Received 16 March 2017; Revised 15 June 2017; Accepted 20 August 2017; Published 28 September 2017

Academic Editor: Xiaopeng Li

Copyright (C) 2017 S. C. Calvert et al. This is an open access article distributed under the Creative Commons Attribution License, which permits unrestricted use, distribution, and reproduction in any medium, provided the original work is properly cited.

\begin{abstract}
With low-level vehicle automation already available, there is a necessity to estimate its effects on traffic flow, especially if these could be negative. A long gradual transition will occur from manual driving to automated driving, in which many yet unknown traffic flow dynamics will be present. These effects have the potential to increasingly aid or cripple current road networks. In this contribution, we investigate these effects using an empirically calibrated and validated simulation experiment, backed up with findings from literature. We found that low-level automated vehicles in mixed traffic will initially have a small negative effect on traffic flow and road capacities. The experiment further showed that any improvement in traffic flow will only be seen at penetration rates above 70\%. Also, the capacity drop appeared to be slightly higher with the presence of low-level automated vehicles. The experiment further investigated the effect of bottleneck severity and truck shares on traffic flow. Improvements to current traffic models are recommended and should include a greater detail and understanding of driver-vehicle interaction, both in conventional and in mixed traffic flow. Further research into behavioural shifts in driving is also recommended due to limited data and knowledge of these dynamics.
\end{abstract}

\section{Introduction}

Road vehicles have gradually become technologically more advanced throughout the past decades with a focus on advancing vehicle safety and comfort. Although vehicle automation has been on the horizon for just as long [1], it is only since the turn of the century that it has started to find its way into production vehicles. Current low-level automated vehicles have technologies such as (adaptive) cruise control and lane-keeping assistance. These advancements form the start of the era of vehicle automation from vehicles that are human-controlled to vehicles that are (partially) automatically controlled $[2,3]$. With this, safety and comfort remain a focus for vehicle manufacturers; however, improved traffic throughput is also now seen as an achievable goal through (partial) vehicle automation $[4,5]$.

Different definitions for the levels of vehicle automation exist. Arguably, the most used definition is that of the Society of Automotive Engineers (SAE) [7], which defines five levels, with the also commonly used definition by Gasser and Westhoff [8], which defines four levels of automation. These levels are (1) driver assistance, (2) partial automation, (3) high automation, and (4) full automation. The SAE scale has an additional level, "conditional automation," which sits in between partial and high automation. Driver assistance and partial automation are considered low levels of automation in which the driver is still required to monitor the system and to perform certain tasks [7,9]. In this paper, the use of the term "vehicle automation" refers ambiguously to any level of automated technology, while the main focus of the contribution is on the lower levels of automation.

There is no lack of predictions stating that automated vehicles will solve many of the current problems experienced on roads today, such as congestion, traffic accidents, and lost time $[10,11]$. Many of these claims are based outside of the scientific community. However, many similar claims are also made within the community, often based on assumptions and forecasts that lack sufficient validation and realism [12]. This cannot always be faulted, as there is still so little known about the actual real-life consequences of automated vehicles in many situations $[2,3,13]$.

There is a lot of evidence that suggests that automated systems will be able to improve traffic flow in the future with sufficient penetration [14-17] and cooperation [18]. These 
advancements would allow for higher operational capacity and may even allow for a reduction in physical infrastructure [19]. However, in the coming years, there will be a slow transition towards (partial) vehicle automation that may not be as positive for traffic flow and operational road capacity. This has also been identified by some, with it also being stated that the wider effects of driver assistance and partial vehicle automation systems are not yet sufficiently understood [20]. Some mechanisms that cause much of this uncertainty are portrayed to the unknown effects in practice. These are the trade-off between safety and efficiency [12, 21], interaction between automated and nonautomated vehicles [2, 22], traffic flow stability [23, 24], and a large number of human factor issues $[12,25,26]$, such as user acceptance and meaningful human control.

While many in science and industry focus on the utopic future of vehicle automation, there will be many years to decades in which there is a real possibility that vehicle automation may have a negative effect on traffic flow [24, 27]. This forms a very real and demanding challenge for road authorities in terms of traffic management and control strategies and in terms of strategic and tactical planning of investment decisions [28]. A significant decrease in road capacity for a vast number of years cannot go unanswered without mitigating action at a cost of millions of dollars or euros of damage to the local economy in delays. On the other hand, expensive expansion and construction of infrastructure also cannot be deemed acceptable if a significant increase in capacity due to vehicle automation could become reality. This forms a difficult balance in strategic decision-making to deal with the effects of vehicle automation, while at the same time so much remains uncertain in relation to what exactly the real effects will be and when and how long they are expected to prevail.

To aid this discussion, this contribution lays out the main foreseen effects of vehicle automation of traffic flow based on a state-of-the-art review of literature and gradually growing understanding of many of the aspects that are expected to influence traffic flow. Further quantification of a number of the effects is estimated in simulation experiments that apply empirically derived characteristics and behaviour of specific types of low-level vehicle automation systems. Application of empirically derived data allows a more realistic estimate, compared to widely applied assumptions. For other effects that cannot yet be realistically modelled due to a lack of behavioural and technological ground truths, further estimations are made of their potential effects based on current advances and best guess estimates.

In the next section, the current state of the art on the effects of traffic flow in mixed traffic is given, especially focusing on vehicle dynamics, traffic flow phenomena, and some additional behavioural aspects. In Section 3, the results of an experimental case are given, in which the effects of lowlevel automated vehicles on traffic flow are estimated for the transition period towards vehicle automation. A discussion of all of the findings from literature and the case is given in Section 4, followed by the main conclusions in Section 5 .

\section{Effects of Automated Vehicles on Traffic Flow in Mixed Traffic}

While the theoretical opportunities of vehicle automation to revolutionise traffic flow are undeniable, they are not expected to be achievable for a number of decades. In the meantime, traffic has already started to undergo a slow transition from human-controlled vehicles to (partially) automated vehicles. Therefore, this section begins with an analysis of the possible time frame in which certain levels of automation may be expected. This is followed by a concise review of literature on the potential effects from changing vehicledriver dynamics and the effects on traffic phenomena. The wider effects of driving behaviour are not explicitly considered in this research, as the quantitative effects on traffic throughput have not been clearly shown yet from research. There are also potential behavioural effects for drivers of conventional vehicles reacting to automated vehicles, which are again insufficiently researched at this time.

2.1. Advancement of Automated Driving. Giving an accurate estimate of the uptake of automated technology is extremely difficult and is heavily dependent on many factors, such as technological development, regulatory incentives or barriers, and economic development $[29,30]$. Furthermore, once a technology is available, it will still take a considerable time before it demands a considerable share of vehicles due to generic market adaption and phasing out of the current vehicle fleet which usually takes approximately 15 years [28]. Many recent attempts have been made to estimate the deployment horizon of automated vehicles. A timeline is given in Figure 1 showing an estimation of the deployment of various levels of vehicle automation in sufficiently large numbers ( $>1 \%$ penetration of vehicle population). This timeline is an estimate based on a wide range of various estimations from academia, industry, and government [27-35]. The figure is by no means meant to be definitive but is meant to give an indication of the duration of the transition phase and what percentages of different automated technologies may be present simultaneously, which is relevant for simulating different types of vehicles.

Based on the cited literature, the vehicle fleet share of automated vehicles will start to significantly increase from 2020 onwards but may still be less than $25 \%$ of all vehicles in 2030. This is not a conservative estimate and shows that the transitional phase from conventional vehicles to automated vehicles is going to be long, lasting many decades [28], which makes it incredibly important to carefully consider. The deployment and share of vehicle cooperation will initially lag behind the penetration rate of automated vehicles but will start to increase from 2025 onwards; however, it will still lag behind due to early automated vehicles remaining uncooperative (note many will be "connected") [13, 19, 34]. For this reason, initial estimates of the effects of vehicle automation should be strongly reluctant to depend too heavily on the presence of any significant share of cooperative vehicle technology. It is conceivable that many, if not most, of the automated vehicles will be connected vehicles from an early stage $[15,18]$, which means that they will be able 


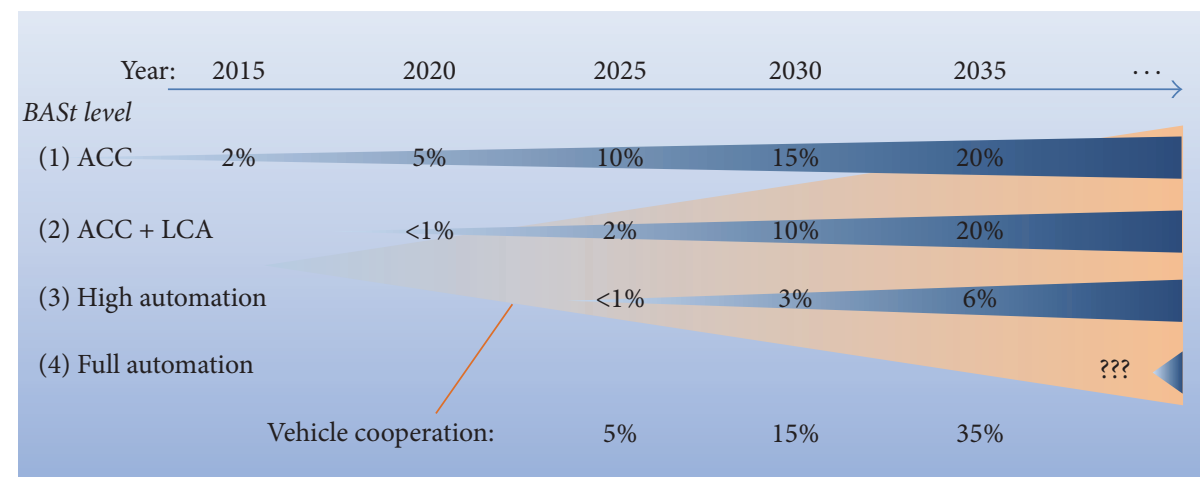

FIGURE 1: Estimated automated vehicle share on roads.

to communicate information, even if that does not mean cooperation. Some difficulties are foreseen for connectivity, due to challenges in protocols, willingness to share data between vehicles from different manufacturers, reliability, and user acceptance $[13,18,36,37]$. Nevertheless, connectivity will make it easier to gradually switch to vehicle cooperation. In this intermediate phase, there will be a varying share of different levels of automated vehicles and conventional vehicles [13]. Traffic will be characterised by human drivers reacting, as they always have, to other conventional vehicles but also needing to consider vehicles that are no longer controlled by a human being. At the same time, vehicles with some level of automation will be present and will also be diverse in capability and technological advancement. These vehicles will need to consider not only conventional vehicles but also actions of other automated vehicles. Vehicle connectivity and cooperation have been rightly hailed as the main requisite to achieve many of the traffic flow and safety gains $[5,18]$. However, cooperative capability and the level of cooperative penetration will initially be too low to be effective and therefore cannot be relied on $[17,18,38]$.

In the following subsection, aspects of this transitional phase are discussed, aided by the current state of the art on these subjects. The main focus in the discussion relates to automation systems and levels of automation which already exist or are currently close to deployment and for which tangible information is available about their effects in mixed conventional-automated traffic and are expected to be present in this phase of deployment. To that extent, the main focus is on driver assistance systems (SAE level 1) and partial automation (SAE level 2), while not disregarding higher levels of automation.

\subsection{Traffic Flow Effects}

2.2.1. Headway. Much research has been performed on the theoretical benefits of vehicle automation on traffic flow, with the majority of the work carried out using microsimulation $[12,14,16,39]$. With respect to systems that control carfollowing behaviour, such as Adaptive Cruise Control (ACC), these approaches may be valid, assuming that the accompanying assumptions are also valid. That, however, is not always the case [12]. The maximum flow of a road, the capacity, is inversely related to the minimum average time headway between vehicles on the road: $q_{\mathrm{cap}}=1 / E\left[t_{\mathrm{hw}}\right]$. In general, a highway is likely to have a capacity between 1800 and 2400 vehicles per hour per lane if only conventional vehicles are present, which translates to operational time headways of on average between 1.5 and 2.0 seconds. In practice, time headways are typically much shorter. This is because the average time headway that governs capacity is a result of a mix of platooning traffic with larger gaps between platoons. Note that when discussing car-following, especially in relation to modelling, one often refers to a desired time gap, which is the time that a driver would like to follow a predecessor at, not including the length of a vehicle. The time headway considers the time difference including the length of the leading vehicle. There is a need to be careful to make a distinction between time headway and time gap and between desired time headway and average time headway (or time gaps), as discussed here.

When considering the longitudinal effect of vehicle automation on operational time headways and time gaps, there are two main things to consider: the actual operational time gap of automated vehicles and their influence on surrounding traffic. The latter is considered later in this subsection in the paragraph on "vehicle dynamics." Experiments carried out in studies on ACC systems have applied varied desired time gaps [40]. Numerous recent studies used time gap settings in a range between 1.0 and 3.0 seconds for the desired time gap of the ACC vehicles, with a main focus on desired time gaps of 1.2-1.8 seconds [14, 16, 17, 20, 26, $30,39,41-43]$. Although these are simulation studies, the authors have derived the values from practice. Desired time gaps of human drivers are thought to generally be in the range of $0.5-1.5$ seconds $[44,45]$. Note again that these are not operational headways that determine capacity but the desired gaps of drivers. This means that the use of ACC in traffic, from a headway point of view, will lead to higher average time headways and therefore to lower capacities. The results of traffic flow effects based on simulations give varying outcomes. Both Calvert et al. [2] and Hoogendoorn et al. [12] suggest that the quality of simulations and their validity are paramount to results being valid. It is questioned to what extent all simulations are sufficiently valid due to a lack of ground truths or use of restrictive models, such as models that 
insufficiently consider driver behaviour. A previous study $[14,15]$ found that a time gap of 1.4 seconds is the critical point between deterioration and improvement of traffic flow although only above a penetration rate of $60 \%$. Others have found vastly varying results from $-5 \%$ to $+10 \%$ on capacity [3], however often applying models that may advantage a more positive outcome due to the mentioned restrictions on realistic driving behaviour. For this reason, a greater amount of empirical evidence is required [2]. It should also be noted that improving technology and string-stability issues also may play a role $[24,46,47]$.

2.2.2. Lane-Changing. Although traffic flow capacity is defined by the longitudinal time headways and time gaps, these are also greatly influenced by lateral movement of vehicles, mainly involving lane-change manoeuvres. When a vehicle changes lane, it requires a sufficient gap in the destination lane and will leave a gap in the origin lane, which requires and results in larger average time headways in the two lanes. Therefore, more lane changes will lead to lower capacities purely based on average time headways. Furthermore, lane changes often lead to other vehicles accelerating or decelerating and therefore increase traffic heterogeneity, which is well known to reduce operational capacity. This is relevant as it has been found that the use of ACC systems generally leads to fewer lane-changing manoeuvres $[6,48$, 49]. There is, however, a possible negative side to this: when lane changes are made, they tend to be performed earlier than a human might make them [50]. Furthermore, Lane Change Assistance (LCA) technology or partially automated vehicles with LCA may generally require a large available gap before changing lanes. While this is alright in free flowing conditions, in busy traffic this may mean that a desired lanechange manoeuvre cannot be carried out or a driver may retake control to manually perform the manoeuvre [43]. It is unclear to what extent these differences in lane-change behaviour will impact traffic flow, as research is lacking in this area at the current time.

2.2.3. Vehicle Dynamics and Interaction. Traffic flow is an interactive and dynamic process in which vehicle interactions play an important role in determining its efficiency. The longitudinal and lateral movements of vehicles are inherently related to vehicle interaction; however, there are more subtle effects of interaction on a vehicular level which play a role. These relate to various stochastic effects and to the level of homogeneity in traffic flow. Disturbances and heterogeneity are known to reduce traffic flow capacity, as larger time headways can appear between vehicles [51, 52]. Human behaviour is inherently stochastic and is wrought with variation on many levels. This is also the case for the driving task; drivers are unable to perfectly maintain a constant speed and following distance, for example, which influences the way vehicles interact. When this stochasticity is applied to traffic flow, it results in heterogeneity in traffic and can be observed in variables such as varying desired speeds between vehicles, at different times, variable gaps, and lateral variations. For many of these dynamic processes, a vehicle with automation technology can perform the same task in a much more stable fashion. For example, when a desired speed is set, the vehicle will hardly deviate from this speed, which has been broadly found from field operation tests $[48,50,53]$. The same is also the case for acceleration and deceleration of a vehicle, which are generally performed much smoother and contribute to a more stable traffic flow [54]. Furthermore, the reaction time with vehicle automation is vastly smaller than for conventional vehicles, which means that disturbances are promptly reacted to, presuming that the time headway is sufficient for traffic to remain string-stable $[55,56]$. At this point, it must be stated that, to achieve the majority of homogeneity gains, a significant penetration of vehicles must be present on the road with longitudinal driving assistance or partial automation $[17,20]$. Due to the requirement of having significant penetration levels, it is not realistic to presume that any major stabilising effect will take place during the transitional period in the coming decade before cooperation is widely available between vehicles. Finally, in relation to vehicle interaction, the time required of vehicles with automated technology to react to other vehicles is much shorter due to substantially shorter reaction times.

2.2.4. Congestion and Propagation. While local capacity and flow conditions are probably the most important aspects to consider for traffic efficiency, other traffic phenomena are also important to consider on a macroscopic scale. Propagation of kinematic waves in traffic is highly relevant for the way local disturbances affect traffic flow along an entire corridor or network. In particular, congestion propagation is of special relevance in this respect. String stability of traffic using a controlled system, such as with ACC, is important and is relevant when considering propagation of kinematic waves in traffic. Most research on string stability is focused on the aggravation or attenuation of disturbances in controlled automated traffic [55]. Experiments have shown that ACC has a limited contribution to the stability of traffic flow $[42,47,57]$ if controllers are set at a string-stable value, often well above 1.0 seconds. For high penetration rates of ACC, say $60-100 \%$, there is evidence that traffic flow will not be stable and will aggravate disturbances [55] and can create and send congestion waves throughout a network. However, as stated previously, such high levels of low-level automated systems are not expected any time soon and when higher penetration rates do appear, the level of automation and, more importantly, vehicle cooperation will be much higher. Both of these developments, especially that of cooperation, have been demonstrated and are widely expected to improve traffic flow stability [47]. In the meantime, there is very little solid evidence on the actual effects of macroscopic traffic flow phenomena for low penetration rates without cooperation and in mixed traffic. Some have generalised the overall effects to be limited and state that low penetration rates will have little effect $[14,58]$. The truth is really that there are too many variables and uncertainties to consider on a traffic microscopic and vehicular level, let alone on a behaviour level, to be able to make accurate statements on the actual traffic kinematic effects at this moment. Traffic modelling has also yet to completely catch up with the advancements [2], 
TABLE 1: Traffic flow effects.

\begin{tabular}{lll}
\hline Aspect & Potential effect & Literature \\
\hline Headway & $\begin{array}{l}\text { Negative (highly dependent on time-headway settings, } \\
\text { driver-use, and stability) }\end{array}$ \\
\hline Lane-changing & Unclear & Small positive \\
(i) Fewer & Small negative possibility & {$[6,43,48-50]$} \\
(ii) Different dynamics & Small positive & Small positive (restricted by low penetration) \\
\hline Vehicle dynamic stochastics & Unknown positive (no evidence) \\
(i) Greater homogeneity & Negligible & {$[48,50,53-56]$} \\
(ii) Shorter reaction time in emergency situations & {$[14,42,47,55,57]$} \\
\hline Congestion and propagation
\end{tabular}

and even with accurate models still too little is known of the applicable parameters.

2.2.5. Summary of Effects. Overall, there are many dynamic processes that will be affected by vehicle automation in traffic flow, of which varying estimates and evidence exist. The initial car-following effects are generally expected to be negative for traffic flow in the transitional phase before expansive cooperation is available due to higher desired time gaps. The effects of lane-changing remain understudied and its total effect may be minimal when considering all aspects involved. The greatest advantage in the transitional phase in traffic flow may be expected to come from a greater degree of homogeneity in traffic. However, these effects may require a higher penetration rate than will be readily expected during 2020-2030. Initially, the effect on other traffic phenomena appears to be limited based on literature, mainly due to the relatively large penetration rates required to have a substantial effect. An overview of the main vehicle related effects is given in Table 1.

\section{Experimental Case}

From the initial analysis, it is clear that there is a potential that there may be negative effects on traffic flow in reality during the transitional phase to vehicle automation. However, there are many uncertainties to this depending on different variables. There are three main areas that can be improved compared to previous (simulation) experiments: the validity of the applied parameter settings, the realism of the applied models, and the inclusion of extensive behavioural aspects of driving. In this experimental case, we will improve on the first two areas, validity and modelling practices, to attempt to answer the hypothesis that "low-level automated systems may have a negative impact on traffic flow." At the moment, insufficient quantitative evidence and modelling methodologies exist to be able to reasonably include the third aspect of accurate and complete driver behaviour in reaction to automated vehicles. Note that driving behaviour will of course be included, just not specifically in relation to the secondary effects described in the previous section. Improvements in validity will be gained through use of data derived from empirical experiments of ACC systems, carried out by Gorter [6]. These experiments give up to date and cross-manufacturer information about the operational use of
ACC in practice. Although the data does not give information on the reaction of other drivers, it does give information on how the ACC driver and vehicle behave. Improvements in modelling are included through the use of improved carfollowing models including stochastic driver behaviour and state-of-the-art lane-changing models.

\subsection{Applied Model}

3.1.1. Integrated Simulation Model. The traffic simulations are carried out using the Lane-change Model with Relaxation and Synchronization (LMRS) [59], which can be integrated with any car-following model. The LMRS incorporates relaxation $[60,61]$, where small headways are accepted during lane changes, and synchronization as a form of lane-change preparation. Consequently, lane changes can occur with small initial deceleration and relaxing headways to normal values. This results in realistic traffic flow characteristics regarding perturbations from lane changes. The integration is formed by the use of the car-following model by the LMRS for gap acceptance and to adjust speed and position for a lane change, which influences car-following parameters. In our case, we use the IDM+ [62] as longitudinal model. IDM+ is an adaptation of the Intelligent Driver Model (IDM) [63]. The IDM has previously been found to be a valid basis for modelling of automated vehicles and extensions have even been implemented in real automated vehicle tests $[16,64$, 65]. The car-following acceleration is determined using (1), where parameter $a$ is the maximum acceleration, $b$ is the maximum comfortable deceleration, $v_{0}$ is the desired speed, $T$ is the desired time gap, and $s_{0}$ is the stopping distance. Furthermore, we have speed $v$, speed difference $\Delta v$, and headway with the leader $s$. Finally, for parameter $\delta$, we use a standard value of 4 , which reduces the maximum acceleration as speed increases.

$$
\begin{aligned}
& v^{\prime}=a \cdot \min \left(1-\left(\frac{v}{v_{0}}\right)^{\delta}, 1-\left(\frac{s}{s^{*}}\right)^{2}\right), \\
& s^{*}=s_{0}+v \cdot T+\frac{v \cdot \Delta v}{2 \sqrt{a \cdot b}} .
\end{aligned}
$$

The LMRS includes lane-change incentives regarding the route, own speed, and keeping to the right lane. Each incentive is included in a normalized lane-change desire 
ranging from -1 to 1 . Different incentives are combined into a single desire to change lane as shown in (2), where $d$ is lanechange desire from one lane to another, $d_{r}$ is the desire from the route incentive, $d_{s}$ is the desire from the speed incentive, and $d_{b}$ is the desire for keeping to the right lane. The latter two, which are voluntary incentives, are included in a factor $\theta_{v}$, which decreases as the absolute mandatory incentive $d_{r}$ increases.

$$
d=d_{r}+\theta_{v} \cdot\left(d_{s}+d_{b}\right)
$$

Depending on the lane-change desire, a few behaviours are assumed to occur. These are divided into four regions of desire as seen in (3). For $d<d_{\text {free }}$, no lane change is performed. For $d_{\text {free }}<d<d_{\text {sync }}$, lane changes are only initiated if they happen to be possible. For $d_{\text {sync }}<d<d_{\text {coop }}$, the subject driver will adjust speed and position relative to the target lane to increase the chance of being able to change lane. Finally, for $d_{\text {coop }}<d$, the potential follower in the target lane will start to cooperate by adjusting speed and position to the subject vehicle.

$$
0<d_{\text {free }}<d_{\text {sync }}<d_{\text {coop }}<1 \text {. }
$$

The level of lane-change desire also affects the accepted deceleration regarding gap acceptance, specifically the threshold given by $d \cdot b$ (where $b$ is the same parameter as in IDM+). Also, the acceptable headway is linearly reduced for increasing lane-change desire, ranging between $T_{\max }$ and $T_{\text {min }}$. Should a lane change be initiated, the desired dependent headway is used from regular car-following and relaxed towards the regular value of $T_{\max }$ exponentially, using a relaxation time $\tau$. The LMRS-IDM+ model in this research is coded in JAVA. For more details on the LMRS and how it is integrated with IDM+, the reader is referred to Schakel et al. [59].

3.1.2. Model Setup. The model is configured using values that have been derived from practice and are therefore feasible real-life values for the variables. Three types of vehicles are defined: regular manual vehicles, low-level automated ACC vehicles (referred to as ACC vehicles from now on), and manual trucks. The regular manual vehicles make use of the standard settings of the IDM+ and LMRS models, as far as these are compatible with the experiment and consistent with the validation data. A similar approach is also used for the manual trucks, whose total modal share is limited in any case. The ACC vehicles are configured differently compared to the manual vehicles, mainly with regard to the longitudinal settings, with a few adjustments also in their lateral lanechange desire. The main variables that differ for the ACC vehicles compared to the manual vehicles are the desired time headway, the desired free flow speed, and the desired lane-change speed difference (i.e., the difference in speed that is required to increase the lane-change desire of a vehicle). Other minor differences are also present but have very limited effect on the model outcome. The minor variable settings are given in Table 2 and the derivation of the desired time headway and desired speeds is explained now in more detail.
TABLE 2: Other relevant vehicles settings used in the model.

\begin{tabular}{lccc}
\hline Variable & $\begin{array}{c}\text { Manual } \\
\text { vehicles }\end{array}$ & $\begin{array}{c}\text { ACC } \\
\text { vehicle }\end{array}$ & Manual truck \\
\hline Maximum speed $[\mathrm{km} / \mathrm{hr}]$ & 200 & 140 & $\begin{array}{c}85 \text { (Gaussian } \\
\text { distribution with } 2.5 \\
\text { standard deviation) }\end{array}$ \\
$\begin{array}{l}\text { Maximum deceleration } \\
{\left[\mathrm{m} / \mathrm{s}^{2}\right]}\end{array}$ & 6.0 & 6.0 & 6.0 \\
$\begin{array}{l}\text { Maximum acceleration } \\
{\left[\mathrm{m} / \mathrm{s}^{2}\right]}\end{array}$ & 1.25 & 2.0 & 0.4 \\
$\begin{array}{l}\text { Lane-change speed } \\
\text { insensitivity }[\mathrm{km} / \mathrm{h}]\end{array}$ & 69.6 & 85 & 69.6 \\
$\begin{array}{l}\text { Minimum lane-change } \\
\text { headway [s] }\end{array}$ & 0.4 & 0.8 & 0.56 \\
\hline
\end{tabular}

In this research, the ACC settings are derived from empirical data, for which we use the work of [6]. In this, empirical time gaps and speeds of vehicle using ACC are measured (see Figure 2). It should be noted that these values are not desired time headways but rather the resulting gaps from the unknown applied settings. A discrete distribution of the desired time headway for the ACC and the manual vehicles is calibrated using the data from Gorter [6] as the resulting distribution. To do this, the distribution from Figure 2(a) is first applied in the model and compared with the output distribution in the model for ACC and manual vehicles. Next, the input distribution is incrementally adjusted until the resulting output is similar to Figures 2(a) and 2(b). The resulting discrete distribution for the input is given in Figures 3(a) and 3(b) and is also applied in the experiment.

From Figure 3, it is clear that there are present values that may not be selectable in most ACC systems. This might raise questions on the validity of the input. However, the difference between the desired time headway settings and the actual time gaps may account for much of this. Furthermore, there is always some discrepancy between model input and real settings in models due to errors that all models make compared to real driving behaviour. Also, a driver's use of ACC and their ability to manually adjust settings and driving behaviour, such as giving extra acceleration, are expected to also be present in the real data, while not being explicitly present in the model. The fact remains that the resulting output of the model using these input values is calibrated and validated against real empirical data and therefore does hold validity to make predictions using these settings. In the following experiment, a further analysis is performed in which the ACC vehicles are given a different distribution for the desired time gap based on an arbitrary range of settings, often seen in other experiments and fitting with the design settings of real ACC systems, which vary from 1.0 to 2.0 seconds $[24,46,66,67]$. This distribution is given by

$$
P(s=\{1.1 ; 1.3 ; 1.5 ; 1.7 ; 1.9\})=0.2 .
$$

Of course, this adjusted distribution is hypothetical and is furthermore not validated using data but does give what we consider to be the upper bounds based on wider literature. The desired free flow speeds for the vehicles are also derived 


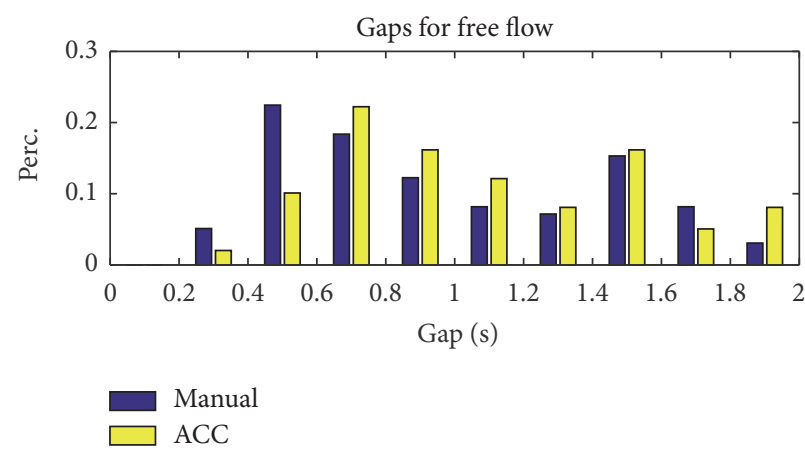

(a)

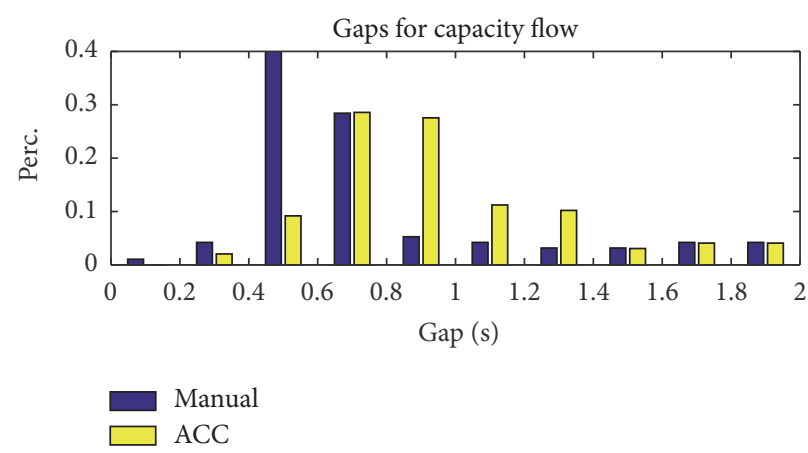

(b)

Figure 2: Vehicle time gaps found adapted from Gorter [6].

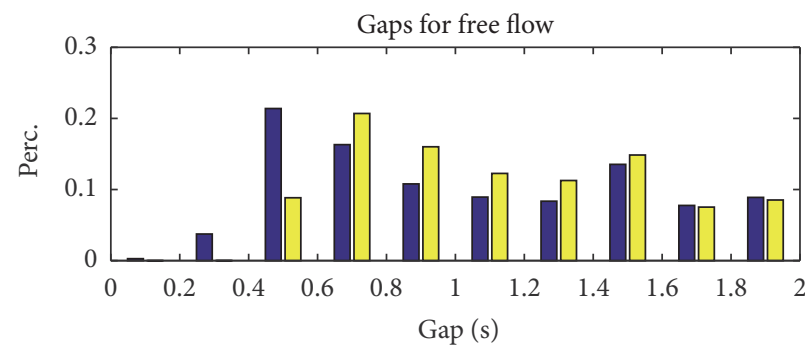

Manual ACC

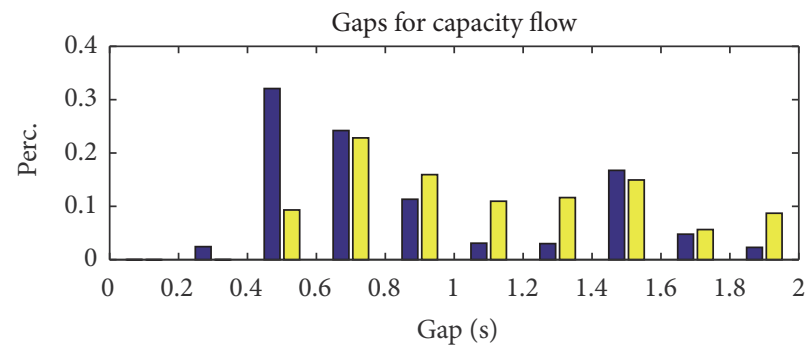

Manual ACC

(a)

(b)

FIGURE 3: Vehicle time gaps calibrated in the model.

from the same work by Gorter [6]. From Gorter's analysis, it became apparent that manual vehicles are generally driven with a distributed speed with a mean just above the speed limit. This also corresponds to the validated standard settings used in the LMRS, and therefore a Gaussian speed distribution for the manual vehicles is derived with a mean of $104 \mathrm{~km} / \mathrm{hr}$ and a standard deviation of $10 \mathrm{~km} / \mathrm{hr}$ for a $100 \mathrm{~km} / \mathrm{hr}$ speed limit. For the ACC vehicles, three distinct peaks were found for their speed settings: one very distinct peak at approximately $104 \mathrm{~km} / \mathrm{hr}$ and two lesser ones at 98 and $100 \mathrm{~km} / \mathrm{hr}$, respectively. Following these findings, the desired speed settings for the ACC vehicles are set at $[98 ; 100$; $104] \mathrm{km} / \mathrm{hr}$ with a probability of $[0.1 ; 0.3 ; 0.6]$. The maximum speeds of passenger cars, both manual and ACC, are vehicle default settings and have no real relevance for the simulation as the desired speed settings are dominant.

\subsection{Case Description}

3.2.1. Road Setup and Traffic Demand. For the experiment, use is made of a simple corridor consisting of a uniform 19kilometre motorway corridor with three lanes and a nominal speed limit of $100 \mathrm{~km} / \mathrm{h}$. After kilometre 16.5, there is an onramp present which acts as a bottleneck, with variable severity depending on the inflow from the bottleneck (see Figure 4). The final 7 kilometres of the corridor are used for qualitative analysis, while the first 12 kilometres are mainly present to comply with good modelling practice by not letting any congestion spill out of the corridor and affect the results of the experiment either by preventing traffic entering the corridor or by biasing the derived travel times. The corridor is shown in Figures 4(a)-4(c), along with the detector location in Figure 4(a), and a demonstration by means of the exploded view and detailed view of traffic flow is given in Figures 4(b) and $4(\mathrm{c})$.

Traffic demand is identical for all scenarios and seeds in the experiment. The experiment is carried out for a 120minute period with an additional 30-minute run-off period, in which no traffic is added, to ensure that the corridor is clear at the end of the simulation. Traffic is released onto the road corridor at an initial rate of $3300 \mathrm{veh} / \mathrm{hr}$ and is slowly increased to $6270 \mathrm{veh} / \mathrm{hr}$ between 10 and 60 minutes to allow capacity to gradually be reached on the road. This level of flow is maintained for 10 minutes and is linearly decreased again at simulation time of 70 minutes towards $0 \mathrm{veh} / \mathrm{hr}$ at time of 120 minutes to allow congestion to dissipate. The simulation is continued for further 30 minutes to ensure that the corridor is empty when finished to allow a fair comparison of all metrics. During the simulation, the inflow rate on the onramp is kept as a percentage of the inflow onto the main corridor. The exact percentage of inflow on the onramp is dependent on the scenario. Therefore, when traffic demand increases on the main corridor, it also increases at the same rate on the onramp. 


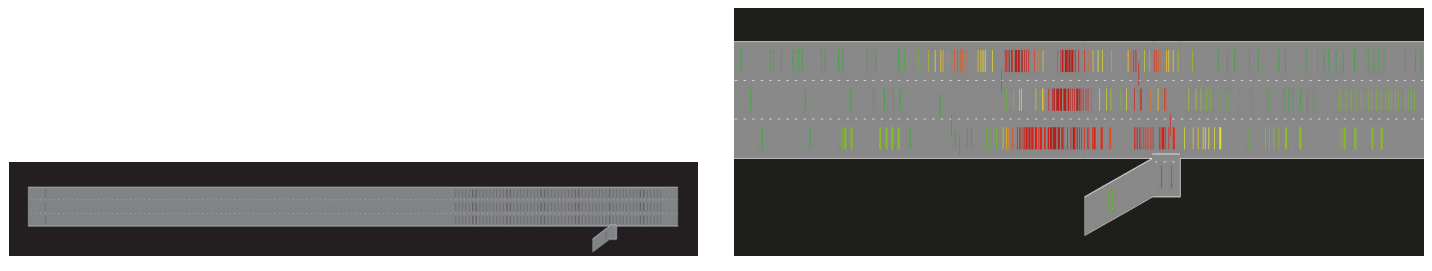

(a)

(b)

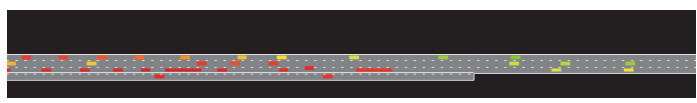

(c)

FiguRE 4: (a) Road corridor for model experiment with detector locations. (b) Exploded view of traffic flow; (c) detailed view of traffic at the bottleneck.

3.2.2. Scenarios. The experiment is set up to test the vehicle dynamic aspects of automated vehicles driving in mixed traffic and therefore considers scenarios in which longitudinal driving, lane-changing, and homogeneity are present to derive their effects on traffic flow. The variables for the experiment scenarios are grouped as follows:

(1) Share of ACC-LKA vehicles using calibrated gap times

(2) Inflow rate from the onramp

(3) Percentage of trucks on road

(4) Share of ACC-LKA vehicles using higher selected gap times

The scenarios that are considered are given in Table 3 and include values that may be expected in the years up to 2030 for ACC share and for an arbitrary onramp flow and truck share. Furthermore, some scenarios make use of higher unlikely levels of ACC vehicles to give an impression of the extremities on traffic flow and for comparison with other literatures. For each scenario, 90 seeded runs are performed to ensure validity and significance of the results.

3.2.3. Performance Indicators. Three performance indicators are used to evaluate the effect on traffic flow from the different scenarios. These are the following:

(1) The breakdown capacity flow, which is defined here as the highest observed aggregated flow during a 5minute simulation period prior to or during traffic breakdown. Traffic breakdown is presumed when the mean traffic speed during the 5-minute period is lower than $70 \mathrm{~km} / \mathrm{hr}$

(2) The discharge capacity flow, which is defined as the highest observed aggregated flow during a 5-minute simulation period out of the congested bottleneck. Again, congestion is presumed when the mean traffic speed during the 5-minute period is lower than $70 \mathrm{~km} / \mathrm{hr}$

(3) Travel time along the entire length of the corridor. This is measured per individual vehicle and grouped by
TABLE 3: Overview of simulated scenarios.

\begin{tabular}{|c|c|}
\hline Scenario group & Applied scenario settings \\
\hline (1) Share of ACC & $\begin{array}{l}\text { Static settings for each scenario: onramp: } \\
18 \% \text { /trucks: } 6 \% \\
\text { ACC gap time settings: calibrated } \\
\text { distribution (see Figure } 3 \text { ) } \\
\text { ACC reference scenarios: } 2 \% \text { (2015), } 12 \% \\
\text { (2025), } 28 \%(2030) \\
\text { ACC scenarios: } 5 \%, 10 \%, 15 \%, 20 \%, 30 \% \text {, } \\
40 \%, 50 \%, 60 \%, 70 \%, 80 \%, 90 \%, 100 \%\end{array}$ \\
\hline (2) Onramp & $\begin{array}{l}\text { Static settings for each scenario: trucks: } 6 \% \\
\text { ACC gap time settings: calibrated } \\
\text { distribution (see Figure } 3 \text { ) } \\
\text { ACC scenarios: } 2 \%, 12 \%, 28 \%, 50 \%, 70 \% \text {, } \\
85 \%, 100 \% \\
\text { For all combinations of onramp: } 14 \%, 18 \% \text {, } \\
22 \%\end{array}$ \\
\hline (3) Truck & $\begin{array}{l}\text { Static settings for each scenario: onramp: } \\
18 \% \\
\text { ACC gap time settings: calibrated } \\
\text { distribution (see Figure } 3 \text { ) } \\
\text { ACC scenarios: } 2 \%, 12 \%, 28 \%, 50 \%, 70 \% \text {, } \\
85 \%, 100 \% \\
\text { For all combination of trucks: } 2 \%, 6 \%, 10 \%\end{array}$ \\
\hline $\begin{array}{l}\text { (4) ACC with higher } \\
\text { gaps }\end{array}$ & $\begin{array}{l}\text { Static settings for each scenario: onramp: } \\
\text { 18\%/trucks: } 6 \% \\
\text { ACC gap time settings: higher nominal } \\
\text { distribution (see (4)) } \\
\text { ACC reference scenarios: } 2 \% \text { (2015), } 12 \% \\
\text { (2025), } 28 \%(2030) \\
\text { ACC scenarios: } 5 \%, 10 \%, 15 \%, 20 \%, 30 \% \text {, } \\
40 \%, 50 \%, 60 \%, 70 \%, 80 \%, 90 \%, 100 \%\end{array}$ \\
\hline
\end{tabular}

time of departure. Therefore this is the actual realised travel time that is collected.

The breakdown capacity allows us to determine the maximum potential flow under the scenario conditions, while the discharge capacity allows comparison with the breakdown to determine possible effects on the capacity drop for the different scenarios. The travel time allows us to evaluate the effects on efficiency throughout all traffic states, from freely flowing traffic through capacity conditions to congested 
traffic. This also captures the efficiency of traffic to transverse resulting congestion shockwaves produced by the bottleneck.

Further qualitative analysis of congestion patterns and onset from speed and flow contour plots allows us to analyse specific traffic flow phenomena and characteristics that may not be as evident from the quantitative analysis.

3.3. Results. In total, 72 scenarios are considered. For all scenario groups, 90 runs per scenario are performed, totaling 6480 in all. After presenting the results, each set of scenarios are discussed for each of the applied performance indicators, and finally an overall comparison is made between scenarios. Visualisation of the three applied metrics for all scenarios is performed with the use of boxplot regression, in which each boxplot is the accumulation of all seeded runs per scenario. The boundaries for the boxplots are the 25th and 75th percentiles, with the whiskers set at a maximum of $150 \%$ of the boxplot range. The results are shown in Figures 5(a)-5(l).

To compare the results of the scenario groups with each other for the influence of ACC share, bottleneck severity (measured by onramp\%), truck share, and ACC gap time, the trends of the means from each scenario are compared in a single figure per performance indicator. This is shown for travel times in Figure 6(a), for the breakdown capacity in Figure 6(b), for the discharge flow in Figure 6(c), and for the capacity drop in Figure 6(d). In each of these figures, a horizontal line indicates the mean value from the base reference scenario of $2 \%$ ACC, $18 \%$ onramp flow, and $6 \%$ trucks, as presumed for the year 2015.

The results are now discussed per scenario group starting with the percentage share of ACC. All results that are discussed are statistically significant at a $99 \%$ confidence interval of the sample mean for the 90 runs per scenario. A greater share of ACC vehicles has a limited negative effect on the travel times. The effect on capacity is a small decrease in capacity for a share of $10-80 \%$ of ACC vehicles of less than $2 \%$ (see Figure 6(b)). Only for a share of above $90 \%$ of ACC vehicles do we see an improvement in traffic flow and road capacity.

The influence of a higher and lower onramp flow gave an unsurprising reduction and increase in capacity and travel times, respectively, as the bottleneck became more severe. The only real outcome of interest for the onramp flow percentage is that, for a low onramp flow, there is no significant deterioration of traffic flow, as seen from higher onramp flows. Further analysis of the results showed that a greater onramp flow forced manual vehicles to perform more lane changes, while for a lower onramp flow fewer additional lane changes were made, which had a positive effect on traffic.

The trend for different traffic shares of trucks also followed an expected trend without any surprises. The results show that a higher truck share leads to lower capacity and longer travel times, while lower truck shares have the opposite effect. There are no further discrepancies in the data to suggest any specific correlation with the ACC vehicle share.

Finally, when the ACC vehicles are set at a higher gap time, there are significantly different outcomes. The travel time trend follows a similar line to the calibrated gap times, only with a high negative deviation and without any eventual improvement at 100\% ACC share. The capacity trend does show a significantly different trend compared to the calibrated gap times; the capacity continues to decrease with increasing ACC share, even for the higher ACC shares. It would seem that the positive effect of more stable traffic with fewer lane changes does not outweigh the larger difference in gap times compared to the manual vehicles. However, the fact that the travel times do improve for the higher ACC shares can be explained when the capacity drop trend is considered. The capacity drop for ACC vehicles with larger time gaps hardly changes for increasing levels of ACC share. For all other scenarios, we see that the capacity drop significantly increases for higher ACC shares in just about equal measure. This increase is not down to a lower discharge flow but rather a higher breakdown capacity with the discharge flow remaining constant. A higher breakdown capacity must be seen as positive; however, a higher capacity drop is bad for travel time reliability. Therefore, the results need to be considered carefully from a policy perspective.

The main findings from the experiments show that, under realistic levels of ACC share, there may be a limited negative effect on both road capacity and traffic flow. For the empirically calibrated gap times, there was a small decrease in capacity of just $1-2 \%$, while for the larger ACC gap times a decrease of $2-7 \%$ was found in capacity. A higher mean gap for ACC vehicles at lower penetration rates is the probable cause, while the advantages of smoother traffic flow only start to occur at higher penetration rates. The experiments further show that there are no substantial effects due to bottleneck severity (in this case, onramp flow) or truck share in relation to the ACC share. The experiment further showed that the negative influence on the discharge flow is marginally greater than on the capacity flow, which in turn results in slightly higher capacity drop values for greater ACC shares. However, the higher capacity drops were only significant for unrealistically high shares of ACC vehicles, certainly higher than the 28\% ACC share estimated in Section 2 for the year 2030. These findings are summarised in Table 4.

3.4. Sensitivity Analysis. To give some indication of the influence of the assumptions made for the static variables in the experiment, an additional sensitivity analysis is performed. This allows us to convey the extent that these variables have on the results and acts as a further face validation of the results. The sensitivity analysis is performed for the following variables: maximum speed, acceleration/deceleration, lanechange speed insensitivity, and lane-change relaxation. The main variables viewed in the experiment do not require any further analysis, as their effect is clear from the experiment results. The analysis is carried out for the 2030-reference scenario (28\% ACC; $18 \%$ onramp flow; $6 \%$ trucks) as a comparison between the ACC settings used in the experiment and the manual vehicles settings. For the analysis, the values for automated vehicles are varied for 30 seeded runs, with the mean and the 25th and 75th percentiles being shown in Figure 7.

The maximum speed settings of the low-level automated vehicles, given in Figure 7(a), show that the maximum speed has a small positively correlated effect on capacity and the 


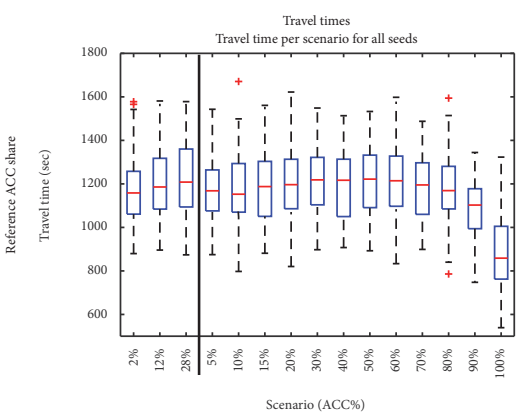

(a)

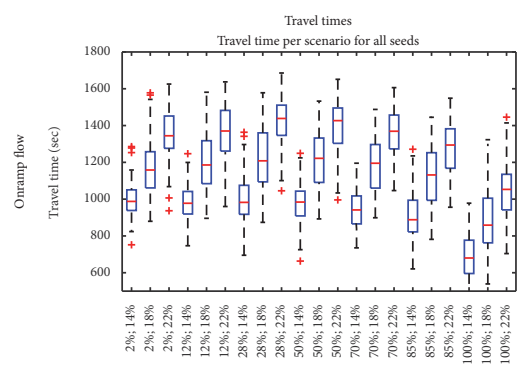

Scenario (ACC-onramp\%

(d)

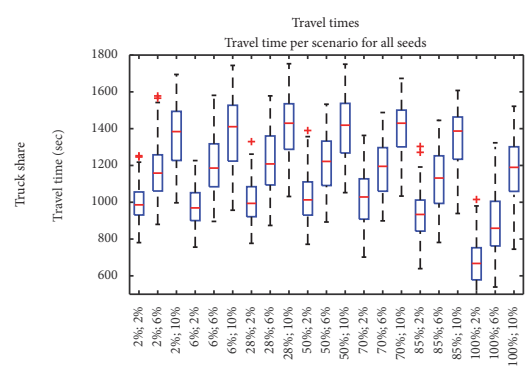

(g)

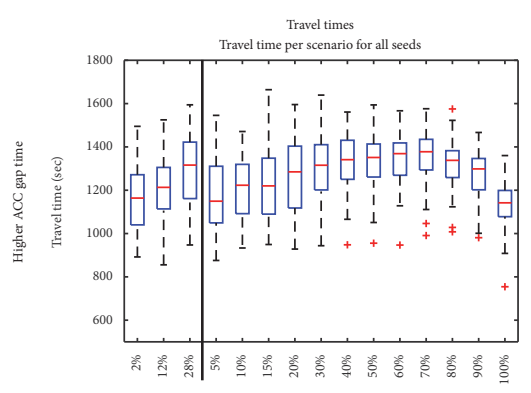

(j)

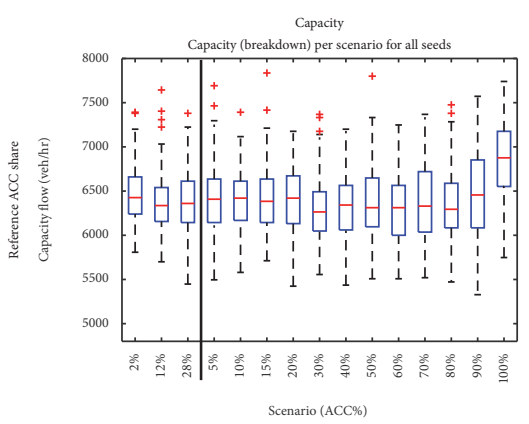

(b)

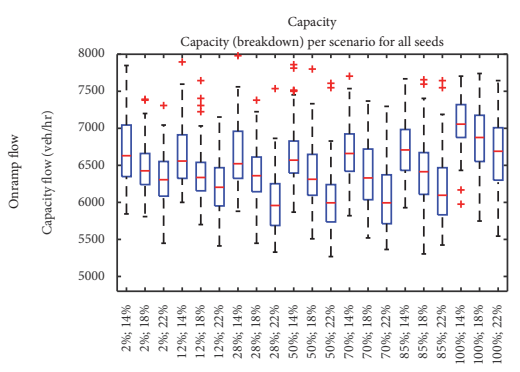

(e)

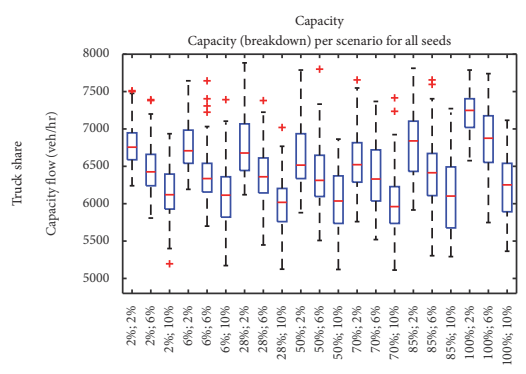

(h)

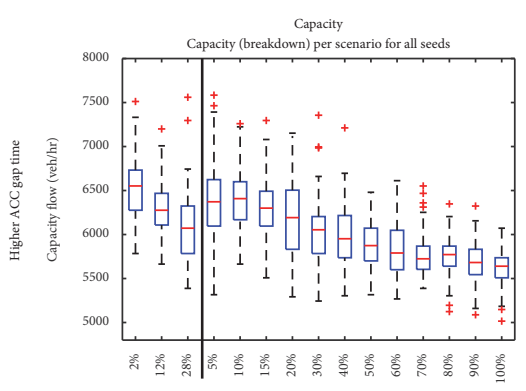

(k)

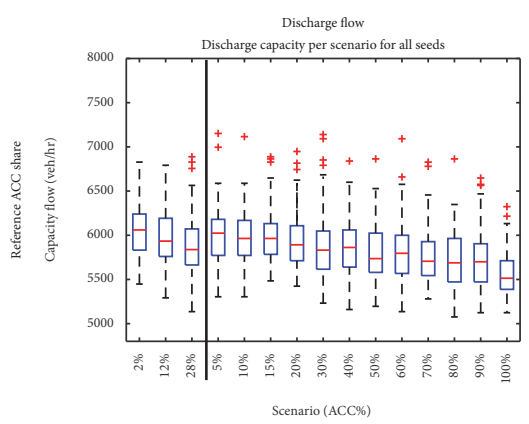

(c)

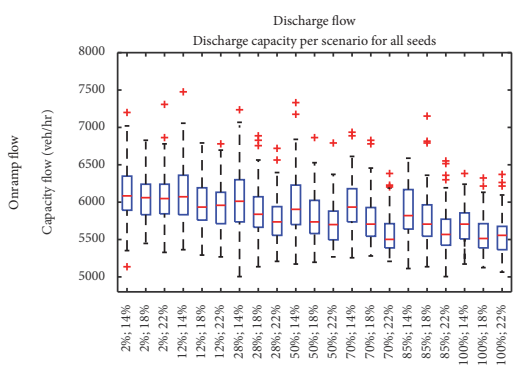

Scenario (ACC-onramp\%)

(f)

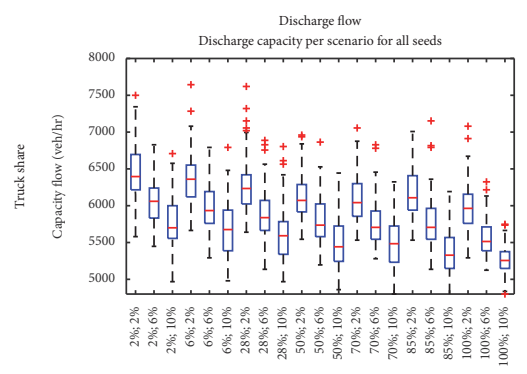

(i)

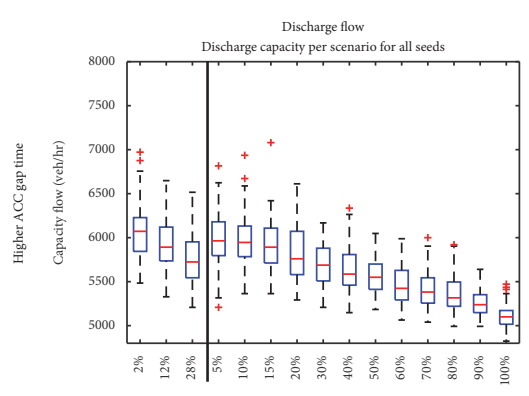

(1)

FIGURE 5: Results of all scenarios for the four scenario groups (vertical) and three performance indicators (horizontal). “+” represents outliers.

opposite for the travel time. This can be expected, especially during the parts of the experiment, when traffic is fluent, as traffic can move at higher speeds. The default value in the experiment for AVs is $140 \mathrm{~km} / \mathrm{hr}$ and $200 \mathrm{~km} / \mathrm{hr}$ for other vehicles. The lane-change desire of vehicles (Figure 7(b)) affects vehicles willingness to voluntarily change lane, with a higher "vGain" value indicating a lower desire. The trend from the analysis shows that variation of the variable has a limited effect on traffic. The effect shown in the figure can possibly be described as negatively correlated to the travel time; however, the results are not significant. The capacity and travel time are shown to be completely insensitive to the maximum acceleration settings of vehicles (Figure $7(\mathrm{c})$ ). The reason behind this is that very few vehicles require, or 

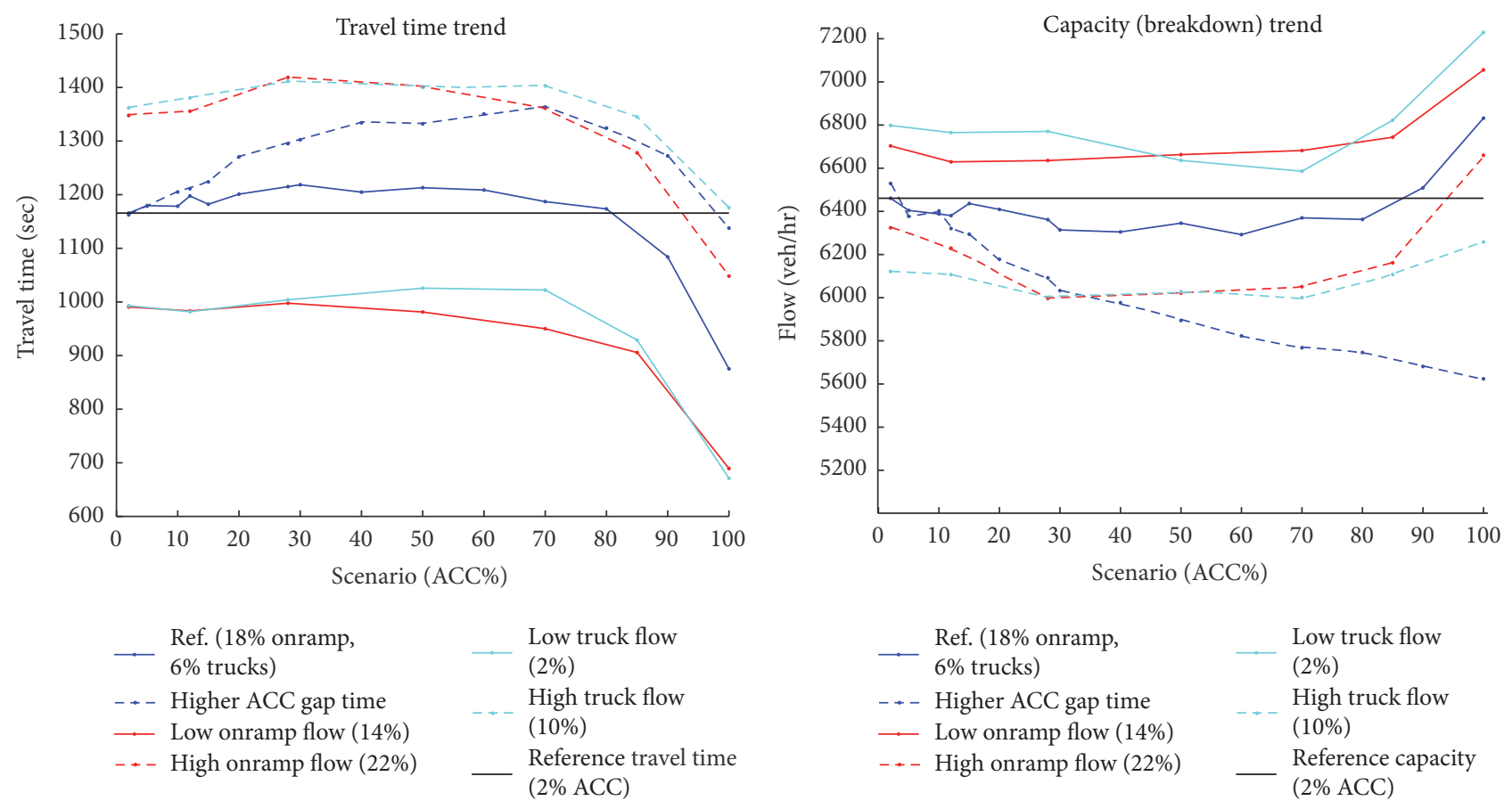

(a) Travel time trend
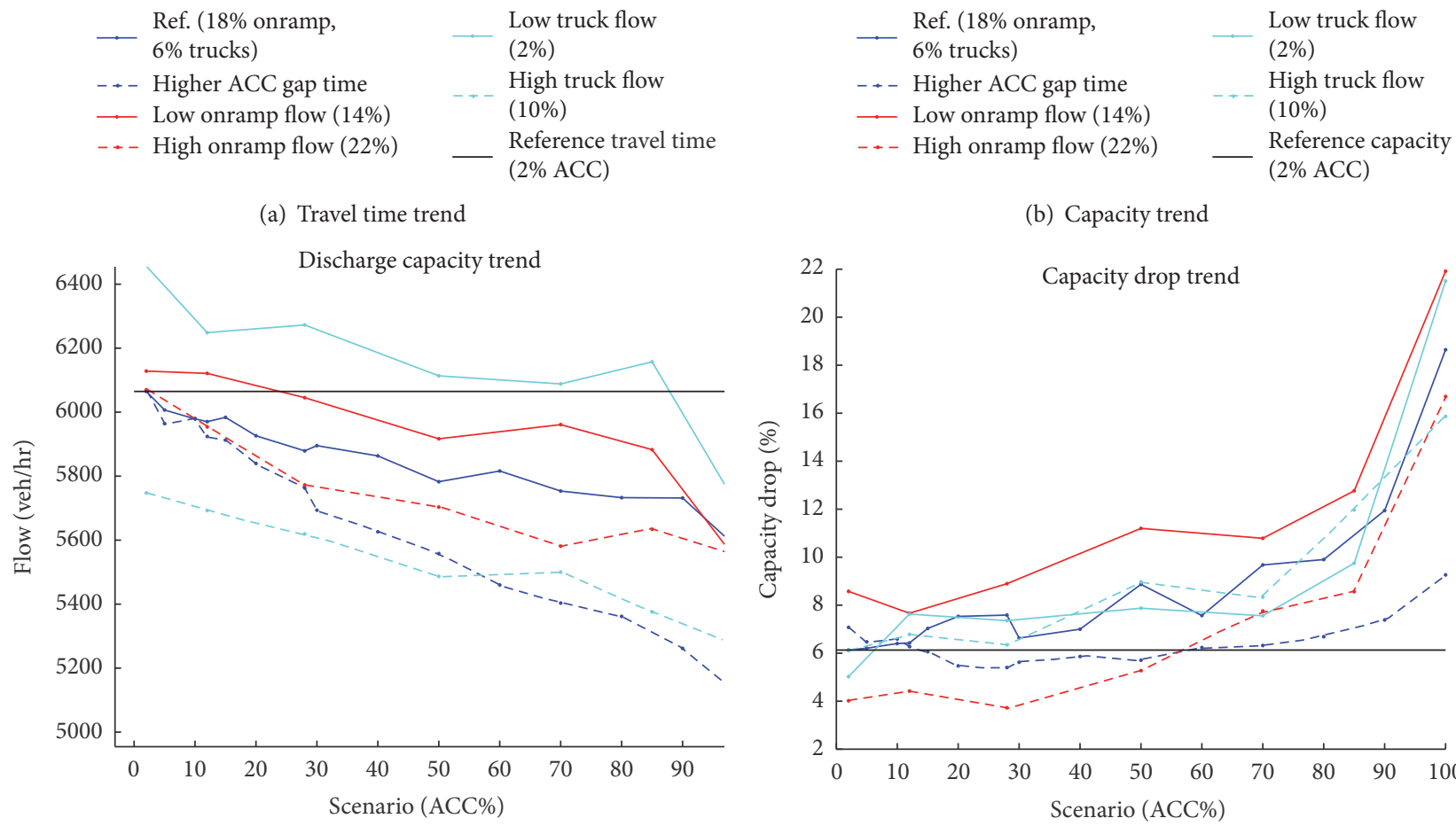

(b) Capacity trend

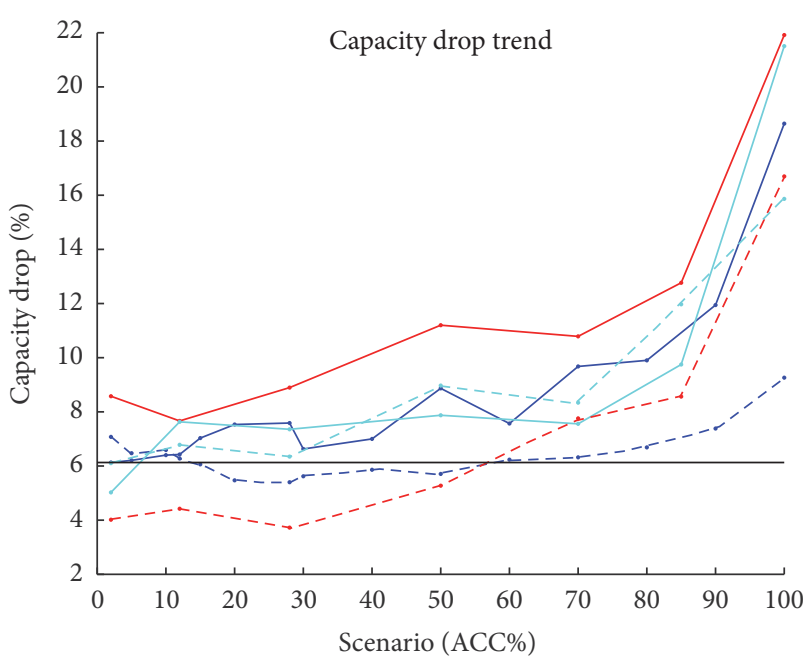

$\begin{array}{lrl}\text { Ref. (18\% onramp, } & -\begin{array}{l}\text { Low truck flow } \\ \text { 6\% trucks) }\end{array} \\ 2 \%) & \begin{array}{l}\text { High truck flow } \\ (10 \%)\end{array} \\ \ldots- & \text { Higher ACC gap time } \\ \ldots-\text { Low onramp flow }(14 \%) & \text { High onramp flow }(22 \%) & \begin{array}{l}\text { Reference flow } \\ (2 \% \text { ACC })\end{array}\end{array}$

(c) Discharge flow trend

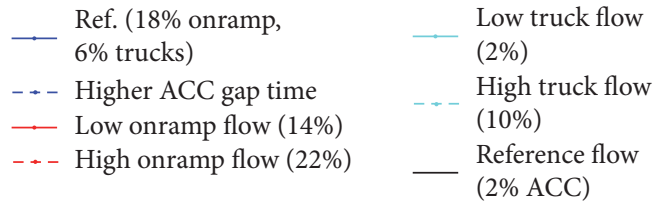

(d) Capacity drop trend

Figure 6

can achieve, acceleration above $1.0 \mathrm{~m} / \mathrm{s}^{2}$ for the considered scenario. Finally, the lane-change relaxation variable is considered, which indicates the gap value that a vehicle can accept in its destination lane for a lane change. Manual vehicle uses 0.4 seconds, with AVs using 0.8 seconds. The results in Figure $7(\mathrm{~d})$ show that there is fair sensitivity to this variable, with lower capacity values for less relaxation (in this case, higher $T_{\min }$ setting) and the opposite trend for the travel time. The effects of the settings of the variables on the experiment and their sensitivity do not through up any surprises. The sensitivities show trends that can be expected and their effects are all plausible. 
TABLE 4: Overall findings from the experiment.

\begin{tabular}{lll}
\hline Aspect & $\begin{array}{l}\text { Found effect of low-level vehicle automation (ACC with } \\
\text { reduced lane-changing) }\end{array}$ & Main possible cause \\
\hline Gap time & $\begin{array}{l}\text { Small negative } \\
\text { Capacity decrease ranging from 1-2\% to 2-7\% } \\
\text { (highly dependent on time-headway settings applied in } \\
\text { the future) }\end{array}$ & $\begin{array}{l}\text { Higher mean gap for ACC vehicles, especially } \\
\text { relevant for low penetration rates }\end{array}$ \\
& $\begin{array}{l}\text { Very small negative } \\
\text { Increase of capacity drop, }<2 \%\end{array}$ & $\begin{array}{l}\text { Lower discharge flow at high penetration rate } \\
\text { due to less aggressive acceleration }\end{array}$ \\
\hline Interaction with trucks & No substantial effect & \\
\hline Bottleneck severity & No substantial effect & \\
\hline
\end{tabular}

\section{Discussion: Dealing with the Traffic Flow Effects of Low-Level Automation}

4.1. Overview of Effects on Traffic. The findings from the experiment and from the review of literature lead us to a number of conclusions with regard to the traffic flow effects of automated vehicles in the initial transitional period. The transition from the current fleet of manual vehicles to the future fleet of vehicles in the coming decades ranging from 2015 to 2035 is expected to be gradual, leading to a combined share of low-level automated vehicles (SAE levels 1 and 2) between 25 and $30 \%$. These vehicles can influence traffic flow through their different behaviour and through the extent of their share of all vehicles. The experiments showed that the relatively low share, of less than 30\% low-level automated vehicles, has a limited effect compared to higher arbitrary penetration levels. Nevertheless, some effects are found and are summarised:

(i) Small negative impact on capacity and flow due to higher gap times

(ii) Marginal increase of capacity drop

(iii) Negligible effect on secondary traffic flow phenomena

These findings should be considered by road authorities, as even small decreases in traffic flow on heavily used roads can have a substantial effect on traffic performance. However, on the other hand, the extent to which negative effects are expected are probably sufficiently limited to not require significant expansion of infrastructure to alleviate the effects. Therefore, we can revisit the hypothesis that "low-level automated systems may have a negative impact on traffic flow" by stating that there is a negative effect; however, the overall effect is limited.

The years following the initial transitional period, probably 2030-2050, are going to be characterised by a strong uptake of vehicle cooperation. Obviously, before 2030, vehicle cooperation may already be present on roads, however at a penetration rate that is too low to make a substantial difference to traffic flow. The positive effects of cooperation have already been well stated, such as increased flow stability and efficiency, and are not explicitly the subject of this paper; however, they do act as a boundary in which the following phase of automation transition in traffic will occur.

There are also other effects on traffic flow as a consequence of low-level automation which are not considered in this research but may also be relevant. Examples are the following:

(i) Effect on user awareness and alertness

(ii) Effect on safety due to quicker reactions times

(iii) Effect on safety due to performing secondary tasks

(iv) User acceptance, particularly in critical situations such as merging

The effects of driver interaction with the system are an intriguing area of research that has yet to lead to conclusive generic effects for traffic flow. Also, safety is indirectly of importance to traffic flow. An estimated 25\% of delays in Netherlands were attributed to incidents on motorways, with similar values found in countries like UK [68]. Improved safety allowing the reduction of these delays can only act to improve traffic performance as a whole.

4.2. Discussion: Modelling and Simulating Automated Vehicles. Simulation of low-level automated vehicles with current simulation models is relatively achievable in many cases through use of current model settings. In fact, one could argue that prevailing traffic simulation models essentially model automated vehicles instead of their human counterparts. This is particularly true for car-following, since in the longitudinal direction drivers are largely constrained by forward system dynamics. The ideal driver model (as well as IDM+ used in this paper) describes an elegant control law that can reproduce most of the longitudinal phenomena we observe in (macroscopic) data, such as the capacity drop, flow instability, and wide moving jams.

The term ideal implies drivers not hampered by typical human behavioural or physiological traits and limitations such as errors in perception, reaction time, risk assessment, and aggressiveness. The same holds for many other carfollowing models, ranging from safe-distance models [69, 70], optimal velocity models [71], or other approaches in the more general group of stimulus-response models [72, 73]. Most "ideal" models can, of course, straightforwardly be augmented to include reaction times, and there are approaches that include more sophisticated mechanisms. Socalled psycho-spacing (or action point) models [74] incorporate drivers' inertia to observe and respond to small changes in stimuli, whereas multianticipatory models $[75,76]$ include terms for anticipation of drivers to traffic conditions further 


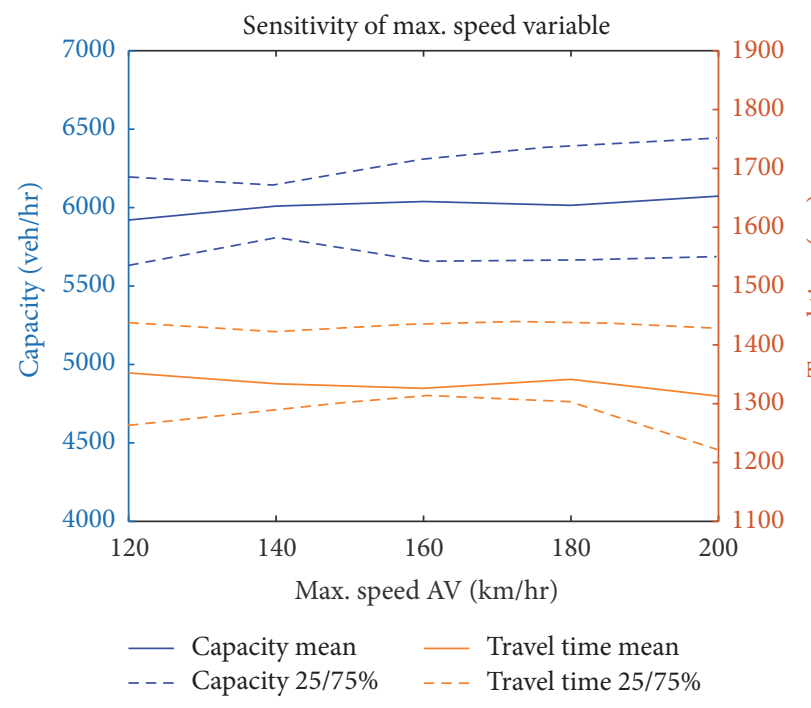

(a) Sensitivity of max. speed

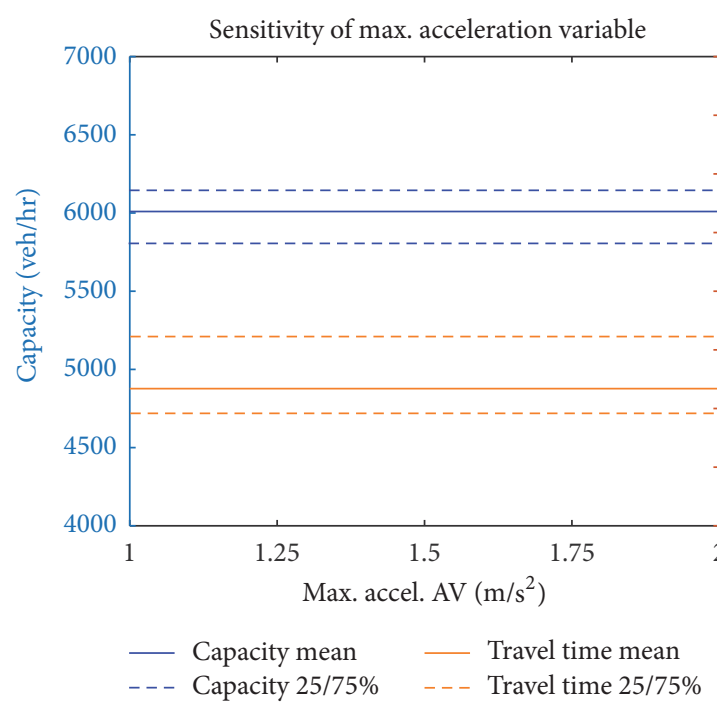

(c) Sensitivity of max. acceleration

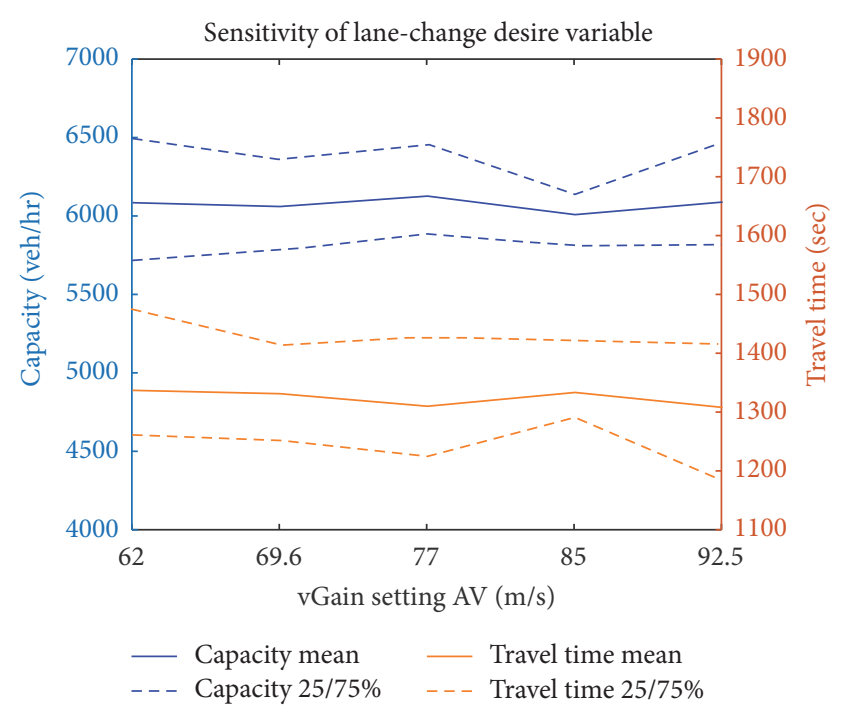

(b) Insensitivity of lane-change speed

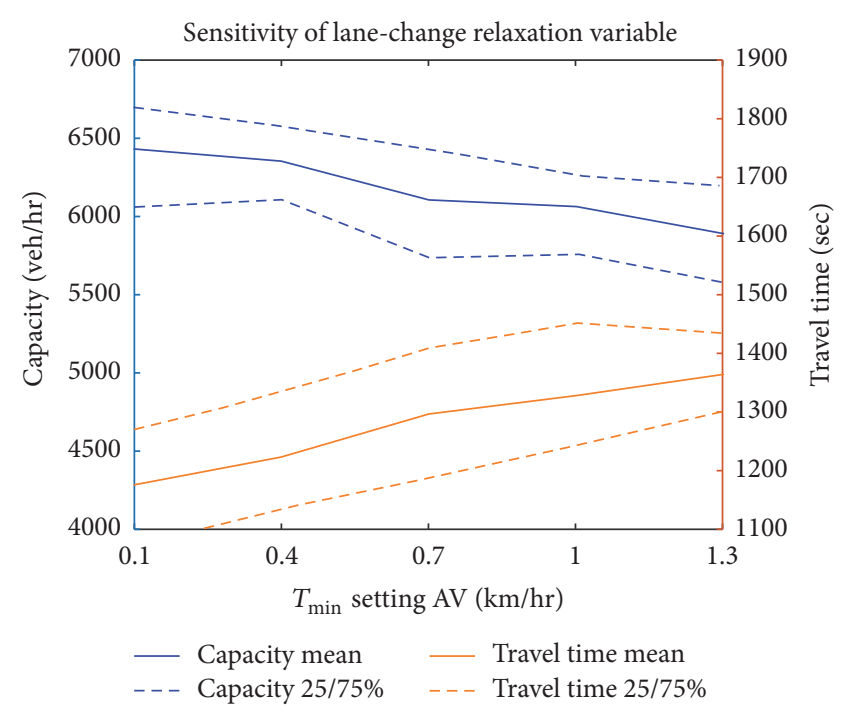

(d) Sensitivity of lane-change relaxation

FIGURE 7

downstream. Other attempts describe more sophisticated human-factor mechanisms. For example, Hamdar and coauthors [77] propose a car-following model based on prospect theory in which drivers increase travel time to prevent the risk of rear-end collisions, and several authors have experimented with Fullers' Task-Capacity-Interface model [78-80]. Incorporating human factors in car-following models remains a very lively research field.

In terms of lateral movement, the discrepancies between what models predict and how human drivers really behave may be even larger. The applied LMRS model in the experiment in this research has a wide range of lane-change and lane-choice parameters and settings that allow for plausible lateral movements at least in terms of the phenomena they reproduce (lane distributions and speed synchronization). But results that seem plausible may not necessarily reflect actual underlying behaviour. Recent empirical research [81] reveals that drivers may follow strategies different from those incorporated in LMRS or any other lane-changing model for that matter. While the LMRS improves on traditional lane-change models by including more strategic lane-change behaviour, there is still some way to go before this behaviour is completely understood and implemented in models. The difficulty clearly lies in the availability of empirical data to validate the lateral movements and choices and to include behavioural changes in that behaviour due to low-level automation in models.

The conclusion must be that simulating longitudinal driving behaviour of automated vehicles may be straightforward; modelling the response of human drivers to these automated vehicles certainly is not. For lateral behaviour, the picture is blurrier, because we lack sufficient empirical evidence to model real human behaviour. The paradox is that most traffic simulation models mimic "ideal" (collision-free) 
human behaviour. Now that vehicle automation becomes a reality, there is a stronger need than ever to incorporate more sophisticated human factors in the underlying models for carfollowing and lane-changing behaviour.

This also means that conclusions drawn from simulations, including the ones in this paper, must be viewed in the light of the (many) assumptions made. This is especially the case for the influence of lateral movement, both in decision-making and in action. Nevertheless, the macroscopic behaviour and calibration of the vehicle behaviour which primarily focused on longitudinal behaviour in this paper still stand and constitute a clear advancement in estimation of the effects of automated driving.

\section{Conclusions}

The gradual introduction of automation technology in vehicles will influence traffic flow in the future; however, there are too many uncertainties to be able to clearly state to what extent. We have summarised many of the common effects that may be expected in the transitional period from manual driving to low-level automated driving and added to the knowledge base with additional findings. This transitional period will be elongated and will probably last well into the 2030s before any significant penetration rate of higher automated vehicles or cooperative vehicles will be present on roads.

The summary, aided with the help of an additional experiment case, gives us reason to believe that low-level automated vehicles in mixed traffic will have a small negative effect on traffic flow and road capacities. The main reason behind the reduction is higher gap times maintained by automated vehicles, while the influence of decreased lane changes does not show significant effects and can only be claimed in theory. The experiment further showed that any improvement in traffic flow will only be seen at penetration rates above $70 \%$, which is far higher than will be the case in reality. The capacity drop also appears to be slightly higher with the presence of automated vehicles; however, large negative effects only occur at the higher unrealistic penetration rates. The experiment also investigated the effect of bottleneck severity and the influence of slower truck shares on traffic flow. Neither of these variables was significantly influenced by the presence of the low-level automated vehicles.

The main focus of the experiment was not on macroscopic traffic flow effects, such as on networks or during an elongated period; however, from literature, it can be argued that there may be a reduction of accidents, which could result in fewer delays. Furthermore, there is also much deliberation on the behavioural effects on drivers and their ability to be able to drive appropriately when faced with a different workload and mental tasks. This is the case for both the drivers of automated vehicles and manual vehicles drivers encountering automated vehicles. For these effects, too little is known to be able to properly quantify the traffic flow effects and this requires many additional researches.

Furthermore, we argue that current knowledge and model development still lack with regard to appropriately capturing much of real driving behaviour, especially in a lateral sense. The applied LMRS-IDM+ model combination offers an improvement in model development but still lacks full implementation of real driver behaviour due to a lack of empirical ground truths and theoretical constructs. A greater effort is required to acquire these behavioural aspects, as well as the previously mentioned cognitive aspects, if traffic simulation models are to be sufficiently enhanced to allow a full comprehensive evaluation of future traffic systems, which involve yet nondeployed vehicle types.

\section{Conflicts of Interest}

The authors declare that there are no conflicts of interest regarding the publication of this paper.

\section{Acknowledgments}

This research is sponsored by the strategic research support programme of Amsterdam Institute of Advanced Metropolitan Solutions (AMS; http://www.ams-institute.org).

\section{References}

[1] S. E. Shladover, "Review of the State of Development of Advanced Vehicle Control Systems (AVCS)," Vehicle System Dynamics, vol. 24, no. 6-7, pp. 551-595, 1995.

[2] S. C. Calvert, A. Soekroella, I. R. Wilmink, and B. v. Arem, "Considering knowledge gaps for automated driving in conventional traffic," in Proceedings of the Fourth International Conference on Advances in Civil, Structural and Environmental Engineering-ACSEE 2016, Rome, Italy, 2016.

[3] D. Milakis, M. Snelder, B. Van Arem, B. Van Wee, and G. H. De Almeida Correia, "Development and transport implications of automated vehicles in the Netherlands: Scenarios for 2030 and 2050," European Journal of Transport and Infrastructure Research, vol. 17, no. 1, pp. 63-85, 2017.

[4] C. Diakaki, M. Papageorgiou, I. Papamichail, and I. Nikolos, "Overview and analysis of Vehicle automation and communication systems from a motorway traffic management perspective," Transportation Research Part A: Policy and Practice, vol. 75, pp. 147-165, 2015.

[5] J. Timmer, L. B. Kool, R. Pel, F. van Est, and Brom., Converging roads: linking self-driving cars to public goals.

[6] M. Gorter, Adaptive Cruise Control in Practice, Delft University of Technology, 2015.

[7] S. O.-R. A. V. S. C. SAE, Taxonomy and definitions for terms related to on-road motor vehicle automated driving systems.

[8] T. M. Gasser and D. Westhoff, "BASt-study: Definitions of automation and legal issues in Germany," in Proceedings of the 2012 Road Vehicle Automation Workshop, 2012.

[9] S. F. Varotto, R. G. Hoogendoorn, B. V. Arem, and S. P. Hoogendoorn, "Empirical longitudinal driving behavior in authority transitions between adaptive cruise control and manual driving," Transportation Research Record, vol. 2489, pp. 105$114,2015$.

[10] T. Litman, "Autonomous Vehicle Implementation Predictions," Victoria Transport Policy Institute, vol. 28, 2014.

[11] A. Hars, "Top misconceptions of autonomous cars and selfdriving vehicles. Thinking outside the box," Innovation Briefs, 2016. 
[12] R. Hoogendoorn, B. Van Arem, and S. Hoogendoorn, "Automated driving, traffic flow efficiency, and human factors," Transportation Research Record, vol. 2422, pp. 113-120, 2014.

[13] R. Bertini, H. Wang, T. Knudson, and K. Carstens, "Preparing a Roadmap for Connected Vehicle/Cooperative Systems Deployment Scenarios: Case Study of the State of Oregon, USA," in Proceedings of the International Symposium on Enhancing Highway Performance, ISEHP 2016, pp. 447-458, deu, June 2016.

[14] J. Vander Werf, S. E. Shladover, M. A. Miller, and N. Kourjanskaia, "Effects of adaptive cruise control systems on highway traffic flow capacity," Transportation Research Record: Journal of the Transportation Research Board, vol. 1800, no. 1, pp. 78-84, 2002.

[15] J. VanderWerf, S. Shladover, and M. A. Miller, "Conceptual development and performance assessment for the deployment staging of advanced vehicle control and safety systems," California Partners for Advanced Transit and Highways (PATH), 2004.

[16] A. Kesting, M. Treiber, M. Schönhof, F. Kranke, and D. Helbing, "Jam-avoiding adaptive cruise control (ACC) and its impact on traffic dynamics," in Traffic and Granular Flow'05, pp. 633-643, Springer, 2007.

[17] S. Shladover, D. Su, and X. Lu, "Impacts of Cooperative Adaptive Cruise Control on Freeway Traffic Flow," Transportation Research Record: Journal of the Transportation Research Board, vol. 2324, pp. 63-70, 2012.

[18] S. E. Shladover, "Cooperative (rather than autonomous) vehicle-highway automation systems," IEEE Intelligent Transportation Systems Magazine, vol. 1, no. 1, pp. 10-19, 2009.

[19] PSC. and CAR., Planning for Connected and Automated Vehicles, Public Sector Consultants (PSC) Center for Automotive Research (CAR), Michigan, USA, 2017.

[20] V. Milanes, S. E. Shladover, J. Spring, C. Nowakowski, H. Kawazoe, and M. Nakamura, "Cooperative adaptive cruise control in real traffic situations," IEEE Transactions on Intelligent Transportation Systems, vol. 15, no. 1, pp. 296-305, 2014.

[21] M. Saffarian, J. C. F. De Winter, and R. Happee, "Automated driving: Human-factors issues and design solutions," in Proceedings of the Human Factors and Ergonomics Society 56th Annual Meeting, HFES 2012, pp. 2296-2300, usa, October 2012.

[22] F. Viti, S. P. Hoogendoorn, T. P. Alkim, and G. Bootsma, "Driving behavior interaction with ACC: Results from a Field Operational Test in the Netherlands," in Proceedings of the 2008 IEEE Intelligent Vehicles Symposium, IV, pp. 745-750, June 2008.

[23] A. Kesting and M. Treiber, "How reaction time, update time, and adaptation time influence the stability of traffic flow," Computer-Aided Civil and Infrastructure Engineering, vol. 23, no. 2, pp. 125-137, 2008.

[24] L. Xiao and F. Gao, "Practical string stability of platoon of adaptive cruise control vehicles," IEEE Transactions on Intelligent Transportation Systems, vol. 12, no. 4, pp. 1184-1194, 2011.

[25] A. H. Jamson, N. Merat, O. M. J. Carsten, and F. C. H. Lai, "Behavioural changes in drivers experiencing highly-automated vehicle control in varying traffic conditions," Transportation Research Part C: Emerging Technologies, vol. 30, pp. 116-125, 2013.

[26] F. A. Mullakkal-Babu, M. Wang, B. Van Arem, and R. Happee, "Design and analysis of full range adaptive cruise control with integrated collision a voidance strategy," in Proceedings of the 19th IEEE International Conference on Intelligent Transportation Systems, ITSC 2016, pp. 308-315, bra, November 2016.
[27] M. Bultmann and A. J. H. Houben, "How autonomous vehicles could relieve or worsen traffic congestion, HERE Deutschland, 2016".

[28] T. Tillema, G. J. v. d. Gelauff, J. Waard, S. Berveling, and Moorman., Paths to a Self-Driving Future, Five Transition Steps Identified, KiM, Netherlands Institute for Transport Policy Analysis, 2017.

[29] J. Svensson, Deployment paths for Vehicle and Road Automation, Support action for Vehicle and Road Automation network.

[30] D. Milakis, M. Snelder, B. Van Arem, B. Van Wee, and G. Correia, Development of automated vehicles in the Netherlands: scenarios for 2030 and 2050, Delft University of Technology, Delft, Netherlands, 2015.

[31] I. Automotive, "Emerging technologies: Autonomous cars-not if, but when," IHS Automotive study, 2014, http://press.ihs.com/ press-release/automotive/self-driving-cars-moving-industrysdrivers-seat.

[32] J. Dokic, B. Müller, and Meyer G., "European roadmap smart systems for automated driving." European Technology Platform on Smart Systems Integration. 2015.

[33] TechSciResearch (2015). Global Autonomous Car Technology Market Forecast and Opportunities, 2035-ADAS, SemiAutonomous, Fully-Autonomous.

[34] J. Zmud, M. T. Tooley, J. Baker, and Wagner., Paths of automated and connected vehicle deployment: Strategic roadmap for state and local transportation agencies, Texas A\&M Transportation Institute, 2015.

[35] P. Gao, H.-W. Kaas, D. Mohr, and D. Wee, Disruptive Trends That Will Transform The Auto Industry, McKinsey \& Company, 2016.

[36] S. E. Shladover, "Progressive deployment steps leading toward an automated highway system," Transportation Research Record: Journal of the Transportation Research Board, vol. 1727, pp. 154$161,2000$.

[37] P. Millot, Designing Human-Machine Cooperation Systems, John Wiley Sons, 2014.

[38] K. Dresner and P. Stone, "A multiagent approach to autonomous intersection management," Journal of Artificial Intelligence Research, vol. 31, pp. 591-656, 2008.

[39] G. Klunder, M. Li, and M. Minderhoud, "Traffic flow impacts of adaptive cruise control deactivation and (Re)activation with cooperative driver behavior," Transportation Research Record, no. 2129 , pp. 145-151, 2009.

[40] M. Huisman, Impacts of (Cooperative) Adaptive Cruise Control Systems on Traffic Flow, Delft University of Technology, 2016.

[41] L. C. Davis, "Effect of adaptive cruise control systems on traffic flow," Physical Review E-Statistical, Nonlinear, and Soft Matter Physics, vol. 69, article 066110, no. 6, 2004.

[42] B. Van Arem, C. J. G. Van Driel, and R. Visser, "The impact of cooperative adaptive cruise control on traffic-flow characteristics," IEEE Transactions on Intelligent Transportation Systems, vol. 7, no. 4, pp. 429-436, 2006.

[43] J. Pauwelussen and P. J. Feenstra, "Driver behavior analysis during ACC activation and deactivation in a real traffic environment," IEEE Transactions on Intelligent Transportation Systems, vol. 11, no. 2, pp. 329-338, 2010.

[44] W. Knospe, L. Santen, A. Schadschneider, and M. Schreckenberg, "Empirical test for cellular automaton models of traffic flow," Physical Review E-Statistical, Nonlinear, and Soft Matter Physics, vol. 70, article 016115, no. 1, 2004.

[45] M. Treiber, A. Kesting, and D. Helbing, "Understanding widely scattered traffic flows, the capacity drop, and platoons as effects 
of variance-driven time gaps," Physical Review E - Statistical, Nonlinear, and Soft Matter Physics, vol. 74, no. 1, Article ID 016123, 2006.

[46] J. Zhou and H. Peng, "Range policy of adaptive cruise control vehicles for improved flow stability and string stability," IEEE Transactions on Intelligent Transportation Systems, vol. 6, no. 2, pp. 229-237, 2005.

[47] J. J. Ploeg, Analysis And Design of Controllers for Cooperative And Automated Driving, Technische Universiteit Eindhoven, 2014.

[48] M. Van Twuijver and M. Pol, "Car owners' experiences with incar speed controlling systems in the Netherlands," in Proceedings of The European Transport Conference (ETC), Strasbourg, France, 2004.

[49] N. Strand, J. Nilsson, I. C. M. Karlsson, and L. Nilsson, "Exploring end-user experiences: self-perceived notions on use of adaptive cruise control systems," IET Intelligent Transport Systems, vol. 5, no. 2, pp. 134-140, 2011.

[50] T. P. Alkim, G. Bootsma, and S. P. Hoogendoorn, "Field operational test "the assisted driver"," in Proceedings of the 2007 IEEE Intelligent Vehicles Symposium, 2007.

[51] E. I. Vlahogianni, M. G. Karlaftis, and J. C. Golias, "Statistical methods for detecting nonlinearity and non-stationarity in univariate short-term time-series of traffic volume," Transportation Research Part C: Emerging Technologies, vol. 14, no. 5, pp. 351367, 2006.

[52] S. C. Calvert, H. Taale, and S. P. Hoogendoorn, "Quantification of motorway capacity variation: Influence of day type specific variation and capacity drop," Journal of Advanced Transportation, vol. 50, no. 4, pp. 570-588, 2016.

[53] G. Marsden, M. McDonald, and M. Brackstone, "Towards an understanding of adaptive cruise control," Transportation Research Part C: Emerging Technologies, vol. 9, no. 1, pp. 33-51, 2001.

[54] M. Hoedemaeker and K. A. Brookhuis, "Behavioural adaptation to driving with an adaptive cruise control (ACC)," Transportation Research Part F: Traffic Psychology and Behaviour, vol. 1, no. 2, pp. 95-106, 1998.

[55] S. Darbha and K. R. Rajagopal, "Intelligent cruise control systems and traffic flow stability," Transportation Research Part C: Emerging Technologies, vol. 7, no. 6, pp. 329-352, 1999.

[56] P. Y. Li and A. Shrivastava, "Traffic flow stability induced by constant time headway policy for adaptive cruise control vehicles," Transportation Research Part C: Emerging Technologies, vol. 10, no. 4, pp. 275-301, 2002.

[57] P. J. Zwaneveld and B. Van Arem, Traffic effects of automated vehicle guidance systems: A literature survey. 1997.

[58] B. Van Arem, J. M. Hogema, C. Vanderschuren, and Verheul., An assessment of the impact of autonomous intelligent cruise control,

[59] W. Schakel, V. Knoop, and B. Van Arem, "Integrated lane change model with relaxation and synchronization," Transportation Research Record, vol. 2316, pp. 47-57, 2012.

[60] S. L. Cohen, "Application of relaxation procedure for lane changing in microscopic simulation models," Transportation Research Record: Journal of the Transportation Research Board, vol. 1883, pp. 50-58, 2004.

[61] J. A. Laval and L. Leclercq, "Microscopic modeling of the relaxation phenomenon using a macroscopic lane-changing model," Transportation Research Part B: Methodological, vol. 42, no. 6, pp. 511-522, 2008.
[62] W. J. Schakel, B. Van Arem, and B. D. Netten, "Effects of cooperative adaptive cruise control on traffic flow stability," in Proceedings of the 13th International IEEE Conference on Intelligent Transportation Systems, ITSC 2010, pp. 759-764, prt, September 2010.

[63] M. Treiber, A. Hennecke, and D. Helbing, "Congested traffic states in empirical observations and microscopic simulations," Physical Review E, vol. 62, no. 2, pp. 1805-1824, 2000.

[64] F. Kranke, H. Poppe, M. Treiber, and A. Kesting, "Driver assistance systems for the active congestion avoidance in road traffic," VDI Berichte, vol. 22, pp. 375-391, 2006.

[65] F. Kranke and H. Poppe, "Traffic guard-Merging sensor data and $\mathrm{C} 2 \mathrm{I} / \mathrm{C} 2 \mathrm{C}$ information for proactive, congestion avoiding driver assistance systems," in Proceedings of the 32nd FISITA World Automotive Congress 2008, 2008.

[66] Acura, "Adaptive Cruise Control (ACC) with Low Speed Follow," User Manual, 2017, http://m.acura.com/pdf/owners/2017/ MDX/2017_MDX_Adaptive_Cruise_Control_with_Low-Speed Follow.pdf.

[67] Toyota, "Toyota automatic highway driving assist," 2017, https://www.toyota-europe.com/world-of-toyota/safety-technology/toyota-automatic-highway-driving-assist.

[68] N. Lazeron and R. van Dinteren, Brein@Work, Bohn Stafleu van Loghum, Houten, 2010.

[69] L. A. Pipes, "An operational analysis of traffic dynamics," Journal of Applied Physics, vol. 24, no. 3, pp. 274-281, 1953.

[70] J. A. Laval and L. Leclercq, "A mechanism to describe the formation and propagation of stop-and-go waves in congested freeway traffic," Philosophical Transactions of the Royal Society of London. Series A. Mathematical, Physical and Engineering Sciences, vol. 368, no. 1928, pp. 4519-4541, 2010.

[71] M. Bando, K. Hasebe, K. Nakanishi, and A. Nakayama, "Analysis of optimal velocity model with explicit delay," Physical Review Letters E, vol. 58, no. 5, pp. 5429-5435, 1998.

[72] D. C. Gazis, R. Herman, and R. W. Rothery, "Nonlinear followthe-leader models of traffic flow," Operations Research, vol. 9, no. 4, pp. 545-567, 1961.

[73] B. S. Kerner and S. L. Klenov, "Deterministic microscopic threephase traffic flow models," Journal of Physics A: Mathematical and General, vol. 39, no. 8, 2006.

[74] Wiedemann, R. Simulation des Strassenverkehrsflusses. Kalsruhe, Traffic Engineering, University of Karlsruhe. 1974.

[75] S. P. Hoogendoorn, S. Ossen, and M. Schreuder, "Empirics of multianticipative car-following behavior," Transportation Research Record: Journal of the Transportation Research Board, vol. 1965, no. 1, pp. 112-120, 2006.

[76] S. P. Hoogendoorn, S. Ossen, and M. Schreuder, "Properties of a microscopic heterogeneous multi-anticipative traffic flow model," in Transportation and Traffic Theory, R. E. Allsop, M. G. H. Bell, and B. G. Heydecker, Eds., pp. 584-606, Elsevier Ltd., London, UK, 2007.

[77] S. H. Hamdar, H. S. Mahmassani, and M. Treiber, "From behavioral psychology to acceleration modeling: Calibration, validation, and exploration of drivers' cognitive and safety parameters in a risk-taking environment," Transportation Research Part B: Methodological, vol. 78, pp. 32-53, 2015.

[78] R. G. Hoogendoorn, B. Van Arem, S. P. Hoogendoorn, and K. A. Brookhuis, "Applying the task-capability-interface model to the intelligent driver model in relation to complexity," in Proceedings of the 92nd Transportation Research Board Annual Meeting, N. Academies, Wash, USA. 
[79] M. Saifuzzaman and Z. Zheng, "Incorporating human-factors in car-following models: a review of recent developments and research needs," Transportation Research Part C: Emerging Technologies, vol. 48, pp. 379-403, 2014.

[80] Z. Zheng, "Recent developments and research needs in modeling lane changing," Transportation Research Part B: Methodological, vol. 60, pp. 16-32, 2014.

[81] M. Keyvan-Ekbatani, V. L. Knoop, and W. Daamen, "Categorization of the lane change decision process on freeways," Transportation Research Part C: Emerging Technologies, vol. 69, pp. 515-526, 2016. 


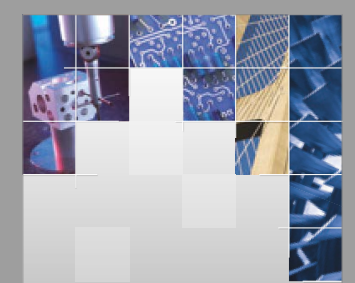

\section{Enfincering}
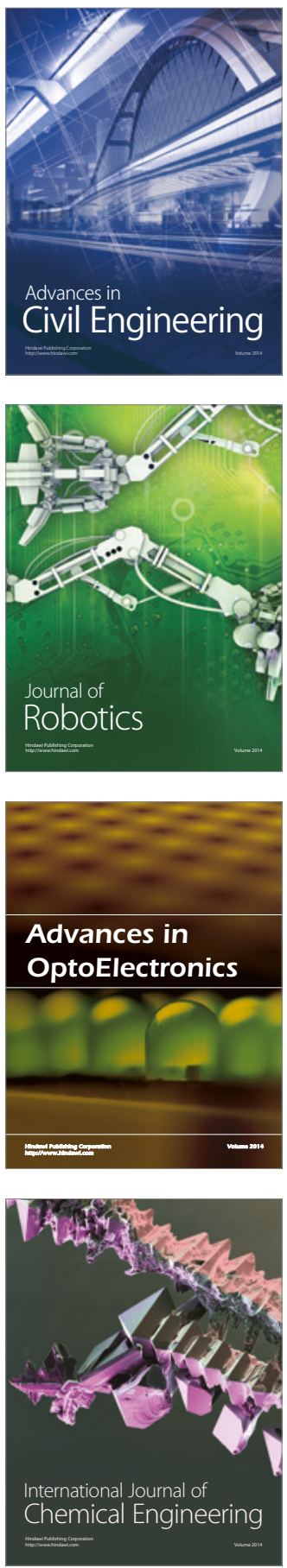

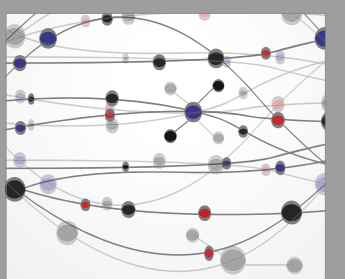

The Scientific World Journal

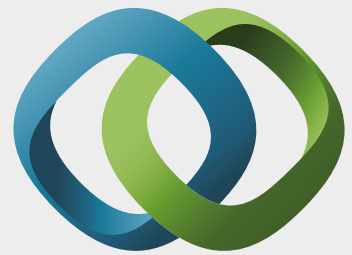

\section{Hindawi}

Submit your manuscripts at

https://www.hindawi.com
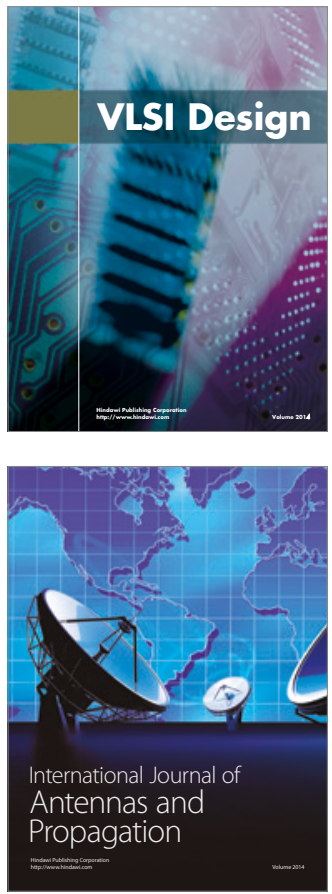

\section{Rotating}

Machinery
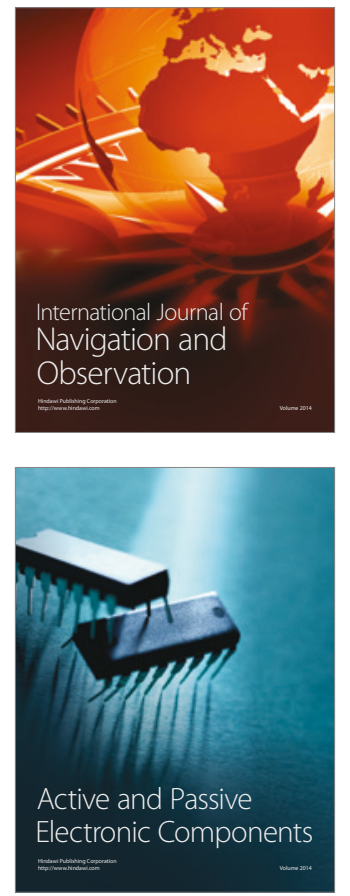
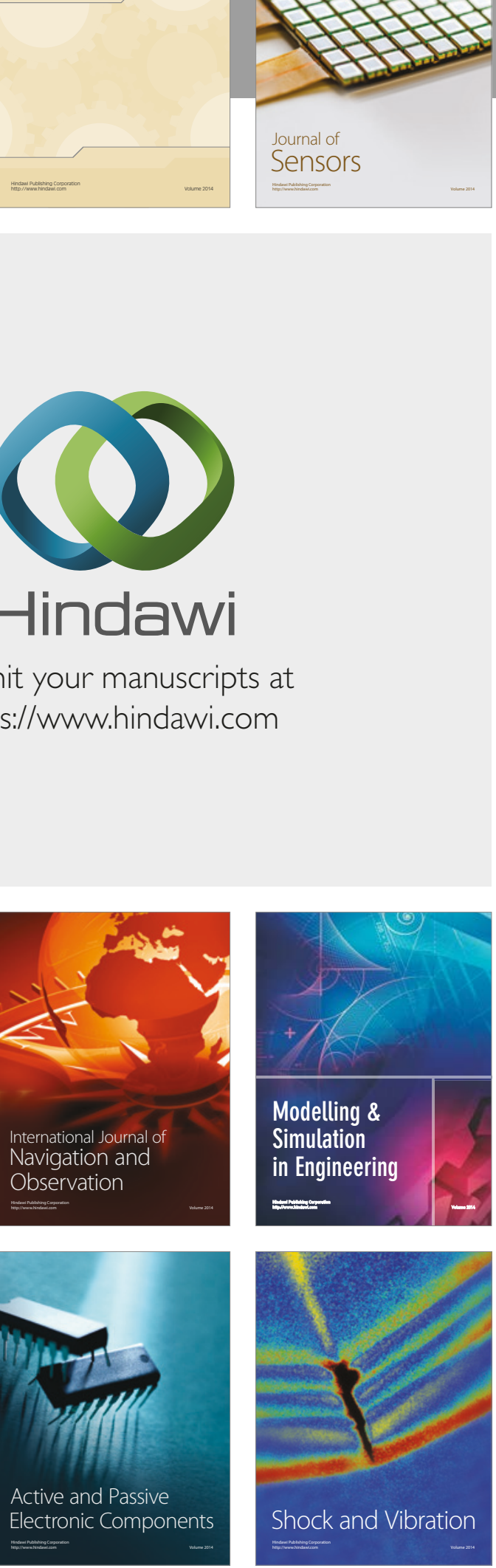
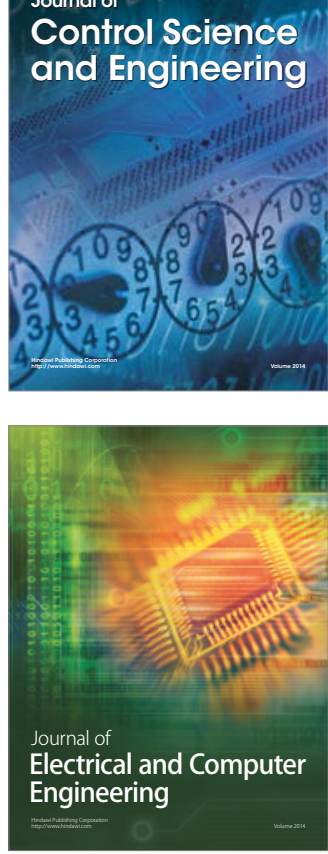

Distributed

Journal of

Control Science

and Engineering
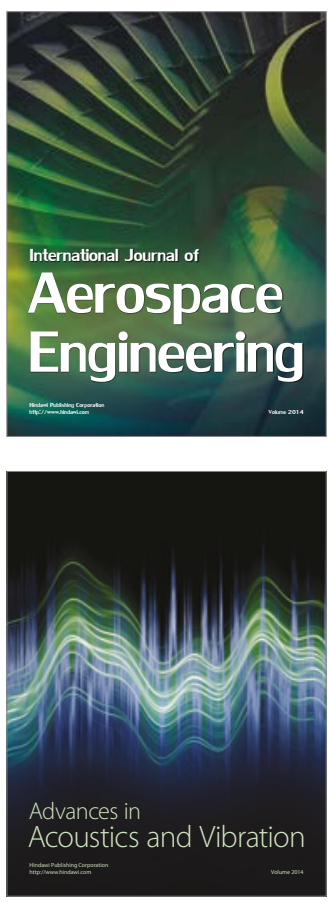

Sensor Networks 